\title{
CFTR- $\beta$-catenin interaction regulates mouse embryonic stem cell differentiation and embryonic development
}

\author{
Zhenqing Liu' ${ }^{1}$, Jinghui Guo ${ }^{1}$, Yan Wang ${ }^{1}$, Zhihui Weng ${ }^{1}$, Biao Huang ${ }^{1}$, Mei-Kuen Yu' ${ }^{1}$ Xiaohu Zhang ${ }^{1,2}$, Ping Yuan ${ }^{3}$, Hui Zhao ${ }^{4,5}$, \\ Wai-Yee Chan ${ }^{4,5}$, Xiaohua Jiang ${ }^{\star, 1,4,5}$ and Hsiao-Chang Chan ${ }^{\star, 1,2,4,5}$
}

Cystic fibrosis transmembrane conductance regulator (CFTR) is a cAMP-regulated anion channel capable of conducting both $\mathrm{Cl}^{-}$and $\mathrm{HCO}_{3}^{-}$, mutations of which cause cystic fibrosis (CF), a common autosomal recessive disease. Although CF patients are known to have varied degree of developmental problems, the biological role of CFTR in embryonic development remains elusive. Here, we show that CFTR is functionally expressed in mouse ESCs. CFTR ${ }^{-1-}$ mESCs exhibit dramatic defect in mesendoderm differentiation. In addition, CFTR physically interacts with $\beta$-catenin, defect of which leads to premature degradation of $\beta$-catenin and suppressed activation of $\beta$-catenin signaling. Furthermore, knockdown of CFTR retards the early development of Xenopus laevis with impaired mesoderm/endoderm differentiation and $\beta$-catenin signaling. Our study reveals a previously undefined role of CFTR in controlling ESC differentiation and early embryonic development via its interaction with $\beta$-catenin, and provides novel insights into the understanding of embryonic development.

Cell Death and Differentiation (2017) 24, 98-110; doi:10.1038/cdd.2016.118; published online 11 November 2016

Cystic fibrosis (CF) transmembrane conductance regulator (CFTR) is a CAMP-regulated anion channel capable of conducting both $\mathrm{Cl}^{-}$and $\mathrm{HCO}_{3}^{-1}{ }^{1}{ }^{1}$ Mutations in the gene encoding CFTR cause cystic fibrosis (CF), a common autosomal recessive disease in Caucasian populations with a wide range of clinical manifestations, such as progressive pulmonary failure, meconium ileus and pancreatic insufficiency. ${ }^{1,2}$ Although CF patients are known to have varied degree of developmental problems in the lung, pancreas, bone and reproductive tract, ${ }^{3-6}$ the biological role of CFTR in development is unknown. It has been observed that CFTR-defective mice have developmental abnormalities, such as low birth weight and severe growth retardation., Moreover, in human embryos, CFTR protein is expressed from the 8-cell stage and functions as a cAMP-regulated apical membrane $\mathrm{Cl}^{-}$channel, reaching maximal expression in the early blastocyst stage. ${ }^{9,10}$ Immunofluorescent staining of the blastocysts clearly demonstrates that CFTR staining is predominant in the inner cell mass of the blastocysts, ${ }^{9,11}$ indicating its potential role in embryonic stem cells (ESCs) function. However, the exact role of CFTR in ESC function and early embryonic development is not known.

Several exogenous factors involved in nucleus-directed signaling pathways, including Lif, Bmp4 and Wnts, have been implicated in modulating ESCs function. ${ }^{12}$ Among them, the Wnt/ $\beta$-catenin signaling pathway which has a central and conserved role in controlling cell proliferation and lineage specification during early embryogenesis, ${ }^{13,14}$ has been shown to cause strong effects on ESCs either in terms of stimulating the expansion of stem cells or promoting differentiation. ${ }^{15-21}$ In mouse ESCs (mESCs), it is generally recognized that the $\mathrm{Wnt} / \beta$-catenin signaling is not required for the establishment of pluripotency, but does help mESCs selfrenewal. ${ }^{18,19,22,23}$ On the other hand, the $\mathrm{Wnt} / \beta$-catenin signaling is critical for mESCs differentiation into mesendoderm (ME), which has the ability to further differentiate into mesoderm and endoderm. ${ }^{24}$ In particular, canonical Wnts, such as Wnt3 and Wnt8a, are necessary for ME differentiation. ${ }^{25,26}$ In contrast, interference of Wnt signaling by the antagonist DKK1 or the small-molecule IWR1-endo dramatically decreases the efficiency of ME differentiation. ${ }^{20,21}$

Of particular interest, a recent study using deep proteomic analysis identified a close association of CFTR with components of Wnt/ $\beta$-catenin pathway among 638 individual highconfidence CFTR interactors. ${ }^{27}$ In addition, our recent studies have demonstrated that CFTR does not merely act as an ion channel, but also mediates different protein-protein

\footnotetext{
${ }^{1}$ Epithelial Cell Biology Research Center, The Chinese University of Hong Kong, Hong Kong SAR, PR China; ${ }^{2}$ Sichuan University-The Chinese University of Hong Kong Joint Laboratory for Reproductive Medicine, West China Second University Hospital, Chengdu, PR China; ${ }^{3}$ Department of Chemical Pathology, The Chinese University of Hong Kong, Hong Kong SAR, PR China; ${ }^{4}$ Key Laboratory for Regenerative Medicine of the Ministry of Education of China, School of Biomedical Sciences, Faculty of Medicine, The Chinese University of Hong Kong, Hong Kong SAR, PR China and ${ }^{5}$ School of Biomedical Sciences Core Laboratory, Shenzhen Research Institute, The Chinese University of Hong Kong, Shenzhen, PR China

*Corresponding author: HC Chan or X Jiang, Epithelial Cell Biology Research Center, Key Laboratory for Regenerative Medicine of the Ministry of Education of China, School of Biomedical Sciences, The Chinese University of Hong Kong, 420A, LKSIBSB, Area 39, Hong Kong, PR China. Tel: +8 52 3943 6797; Fax: 26037155; E-mail: hsiaocchan@cuhk.edu.hk or xjiang@cuhk.edu.hk

Abbreviations: CFTR, cystic fibrosis transmembrane conductance regulator; CF, cystic fibrosis; ESC, embryonic stem cells; ME, mesendoderm; MQAE, N-ethoxycarbonylmethyl-6-methoxyquinolinium bromide; WT, wild-type; KO, knock-out; EB, embryoid body; MO, morpholino; LIF, leukemia inhibitory factor; Axin, Axis inhibition protein; Ccnd1, cyclin-D1; CHIR99021, mitogen-activated protein kinase inhibitor; ERK, extracellular-signal-regulated kinases; GATA4, GATA-binding protein 4; GATA6, GATA-binding protein 6; GSC, Goosecoid; GSK3 $\beta$, glycogen synthase kinase 3 $\beta$; LEF1, lymphoid enhancer-binding factor 1; SSEA1, stage-specific embryonic antigen-1; T, Brachyury; TCF1, hepatocyte nuclear factor 1 homeobox A

Received 15.7.16; revised 04.9.16; accepted 16.9.16; Edited by P Salomoni; published online 11.11.16
} 
interactions that lead to the activation of cell signaling and cell differentiation. ${ }^{28,29}$ Given the observed developmental abnormalities in CF patients and animals, and the paramount importance of Wnt/ $\beta$-catenin pathway in ESCs and embryonic development, it is tempting for us to speculate that CFTR may regulate ESCs differentiation and embryonic development through the $\mathrm{Wnt} / \beta$-catenin signaling pathway. In the present study, we sought to investigate the role of CFTR in early embryonic development by using CFTR-defective mESCs as an in vitro model and Xenopus laevis as an in vivo model. Our study reveals an interaction between CFTR and $\beta$-catenin, defect of which may affect mesendoderm differentiation and early embryonic development.

\section{Results}

Characterization of CFTR $^{-1-}$ mESC lines. From the animal breeding record, we noticed that the birth rate of homozygote CFTR KO or $\triangle \mathrm{F} 508$ mice was much lower than the predicted by the Mendelian ratio (Supplementary Figure S1a and b), indicating that CFTR defect might have a negative impact on embryo development. We decided to explore the role of CFTR in early embryonic development starting from ESCs. As shown in Figure 1a, CFTR was expressed in undifferentiated mESCs at the mRNA and protein level. Immunofluorescent staining showed that CFTR was localized in the cytoplasm and membrane in mESCs (Figure 1b). Furthermore, intracellular $\mathrm{Cl}^{-}$imaging using a $\mathrm{Cl}^{-}$sensitive dye, MQAE (N-ethoxycarbonylmethyl-6-methoxyquinolinium bromide), showed that intracellular $\mathrm{Cl}^{-}$concentration $\left(\left[\mathrm{Cl}^{-}\right] \mathrm{i}\right)$ was decreased by CFTR activator $(10 \mu \mathrm{m}$ Forskolin) whereas increased by CFTR inhibitor $(10 \mu \mathrm{m}$ inh172; Figure 1c), indicating that CFTR is functional and medicating $\mathrm{Cl}^{-}$efflux in mESCs. We also showed that CFTR was expressed and functional in the hESC line, $\mathrm{H} 9$ as well (Supplementary Figure S2a and b). To further study the function of CFTR in mESCs, we crossed the CFTR $^{\text {tm1unc }} \pm$ mice $^{4}$ to establish CFTR wild-type and knockout (KO) mESCs lines (Supplementary Figure S3a and b). With the primers targeted to the adjacent sequence of exon 10 and the neomycin selection cassette, PCR confirmed that transcription of CFTR was stopped in CFTR $^{-1}$ mESCs lines (Supplementary Figure S3c and d). Two pairs of wild-type (WT1 and WT2) and CFTR ${ }^{-1-}$ double-knock-out (KO1 and KO2) mESCs lines were selected for further study. Both CFTR WT and KO cells maintained the typical mESCs morphology under LIF+2i (Figure 1d). Alkaline phosphatase and SSEA1 staining (indicator of ESCs stemness) of CFTR WT and KO mESCs revealed that CFTR deficiency resulted in mild reduction of self-renewal capacity (Figure 1e, $f$ and Supplementary Figure 3e). Western blot analysis further confirmed that the expression levels of pluripotency markers Oct4, Sox2 and Nanog were similar between WT and KO mESCs (Figure 1g) in LIF+2i. Taken together, these data suggest that CFTR may not have a major role in the selfrenewal of mESCs.

CFTR deficiency impairs mesendoderm differentiation of mESCs. We then proceeded to examine the role of CFTR in spontaneous differentiation of mESCs into the embryoid body (EB). We observed that the expression level of CFTR was gradually increased during early EB formation from day 1 to day 7 reaching a maximum at day 4 , suggesting an important role of CFTR in mESC early differentiation (Figure 2a). To further analyze whether or not the CFTR deficiency interferes with lineage-specific differentiation, WT and KO mESCs were cultured without LIF and $2 \mathrm{i}$ to grow EB and induced to differentiate into three primary germ layers for 7 days. Although we did not find any morphological difference between WT and KO EB from day 1 to 7 (Figure 2b), the whole-genome microarray data showed that CFTR deficiency impaired the development process and led to a global downregulation of mesoderm, endoderm and germ cell markers at day 5 (Figure 2c and Supplementary Figure S4). To further evaluate the dynamic regulatory role of CFTR in mESC differentiation, we determined the expression of mesoderm/mesendoderm ( $T$, Mix/1, Gsc and Eomes), endoderm (Gata4, Gata6, Sox17, Foxa2) and ectoderm (Pax6, Fgf5) markers in WT and KO mEBs induced from mESCs at day 1, 2, 4 and 7. As shown in Figure $2 \mathrm{~d}$, the expression of the early mesendoderm markers examined, such as $T$, and Mix/1, was dramatically downregulated in $\mathrm{KO}$ mEBs compared with WT mEBs, starting from day 4. Following that, the expression of mesoderm and endoderm markers (Gsc, Eomes, Gata4, Gata6, Sox17 and Foxa2) was prominently downregulated at day 7 . The expression of ectoderm markers, such as Pax6 and Fgf5, was slightly different between WT and KO mEBs (Figure 2d). As EB differentiation is concomitant with the occurrence of spontaneous apoptosis, we then compared apoptotic response between WT and KO mEBs along differentiation. Annexin V-FITC staining analysis showed that there were more apoptotic cells in KO mEBs than that in WT mEBs (Supplementary Figure S5). Taken together, these results indicate that CFTR deficiency dramatically impairs mesendoderm differentiation in mESCs.

To further evaluate the effect of CFTR deficiency on modulating germ layer differentiation of mESCs in vivo, WT and $\mathrm{KO}$ mESCs were injected into nude mice for teratoma formation. After 4 weeks, teratomas from WT and KO mESCs were collected for HE staining and analysis of germ layer markers. By histological analysis, whereas all three primary germ layer differentiation could be detected in WT and KO mESCs-formed teratomas, more mature mesoderm-derived tissues were identified in WT teratomas than that in $\mathrm{KO}$ teratomas (Figure 3a). Consistent with the EB results, realtime PCR analysis revealed that the expression of primitive streak, mesendoderm and mesoderm markers was significantly repressed in $\mathrm{KO}$ mESCs-formed teratomas (Figure $3 \mathrm{~b}$ ). In addition, western blot results confirmed the downregulation of mesoderm marker a-SMA in KO mESC-formed teratomas (Figure 3c). Collectively, these results further support that CFTR deficiency decreases mesendodermal commitment in vivo.

CFTR deficiency downregulates Wnt/ $\beta$-catenin signaling pathway during differentiation. As $W n t / \beta$-catenin pathway is essential for the mesendoderm differentiation of mESCs, we first compared the expression levels of $\beta$-catenin in WT 


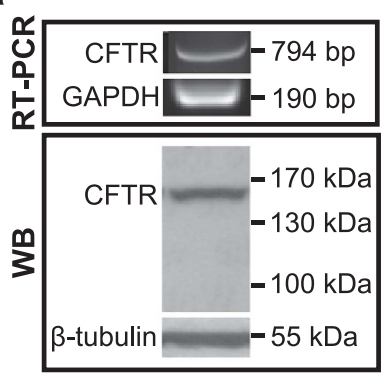

b

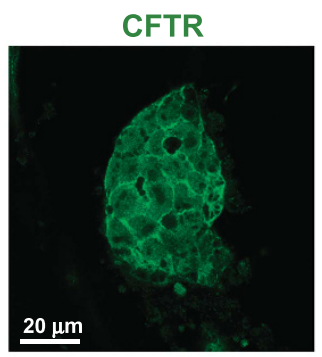

OCT4

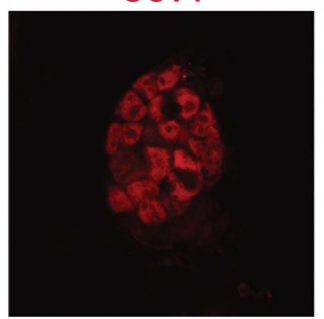

Merge

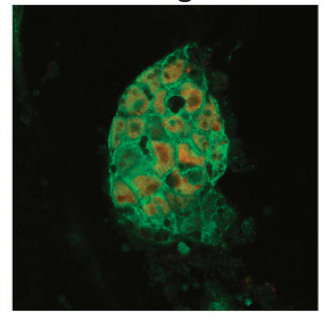

C

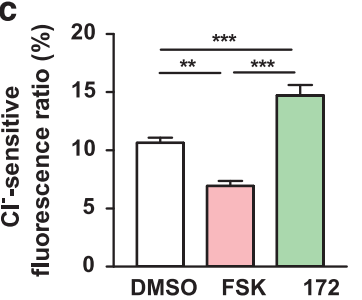

e

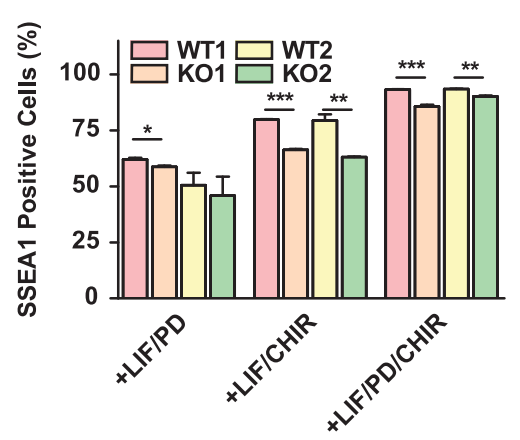

g

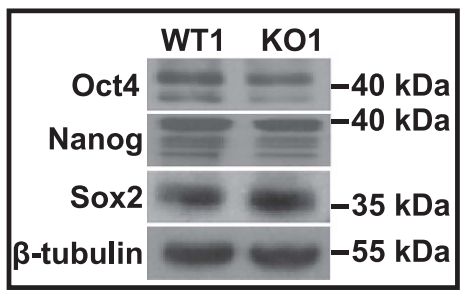

d

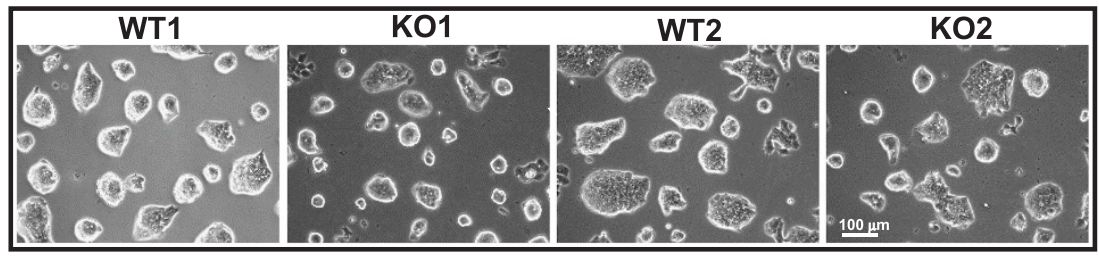

f
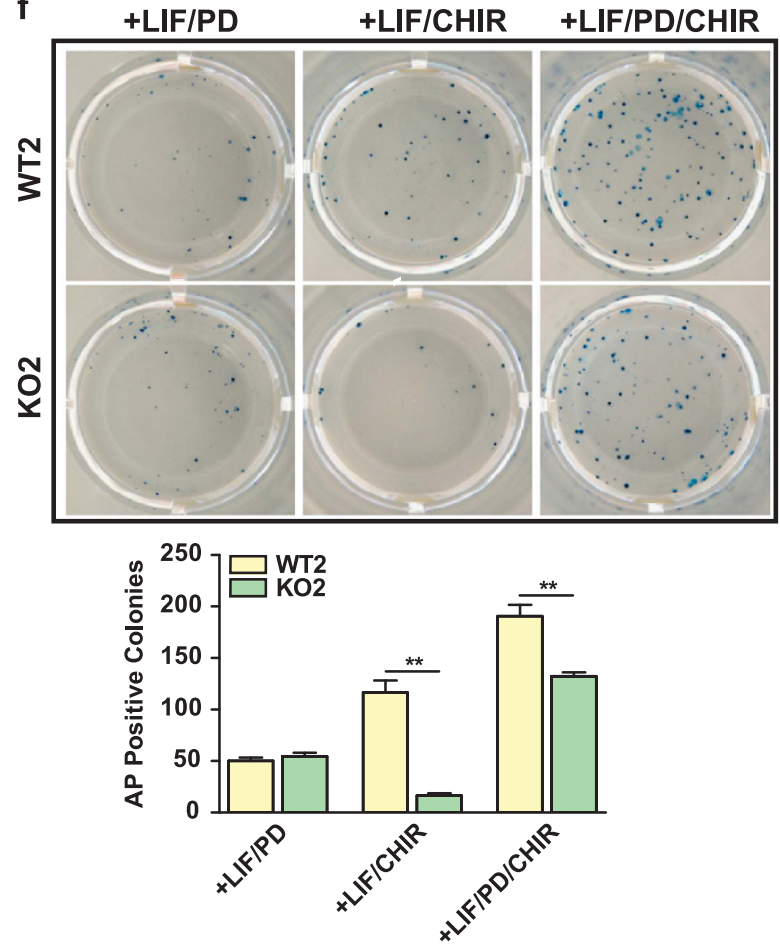

Figure 1 CFTR expression in mESCs and its effect on self-renewal. (a) CFTR is expressed in mESCs; (b) immunofluorescent staining of CFTR (green) and Oct4 (red) in mESCs; (c) percentage changes in $\mathrm{Cl}^{-}$-sensitive MEQ fluorescence intensity in response to CFTR activator, forskolin $(10 \mu \mathrm{M})$ or inhibitor, CFTR inh $^{-172}(10 \mu \mathrm{M})$; (d) phasecontrast images of wild-type and CFTR knock-out mESCs grown in LIF+2i (PD0325901, $1 \mu \mathrm{m}$; CHIR99021, $3 \mu \mathrm{m}$; and LIF (1000 U/ml); (e) Flow Analysis of SSEA1 positive WT and KO mESCs cultured in different conditions (LIF/PD or LIF/CHIR or LIF/CHIR/PD, detailed in the 'Materials and Methods' section); (f) the images and number of alkaline phosphatase-positive colonies in WT1 and KO1 mESCs cultured in different conditions (LIF/PD or LIF/CHIR or LIF/CHIR/PD) for 5 days; (g) western blot analysis of pluripotency markers (Oct4, Nanog and Sox2) in WT and KO mESCs; each bar in the figure represents the mean \pm S.E.M. of triplicates. ${ }^{*} P<0.05,{ }^{* *} P<0.01$ and ${ }^{* \star \star} P<0.001$

Figure 2 CFTR deficiency impairs mesendoderm differentiation of mESCs. (a) Real-time PCR analysis of CFTR mRNA expression in mESCs and developing mEBs from day 1 to day 7; (b) phase-contrast images of WT and KO mEBs at day 1,2, 4 and 7; (c) microarray analysis of day 5 WT and KO mEBs; the heat-map shows all genes with expression level increased or decreased for more than 1.5-fold (left) and three germ layer (endoderm, mesoderm and ectoderm) genes with expression level increased or decreased (right) in CFTR KO mEBs compared with WT mEBs; (d) real-time PCR analysis of mesendoderm, endoderm and ectoderm markers in WTand KO mEBs induced from mESCs at day 1, 2, 4 and 7; quantification data are derived from three independent experiments, ${ }^{*} P<0.05,{ }^{* *} P<0.01$ and ${ }^{* * *} P<0.001$ 
a

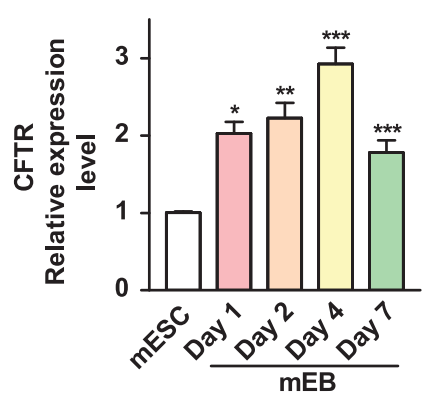

b

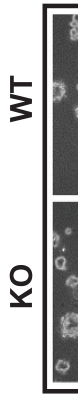

Day1

Day2

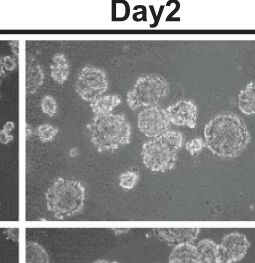

Day 4

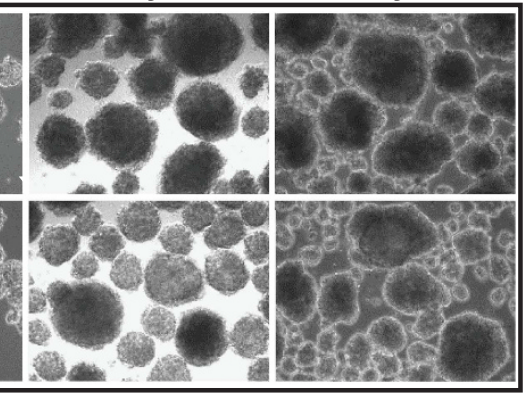

C

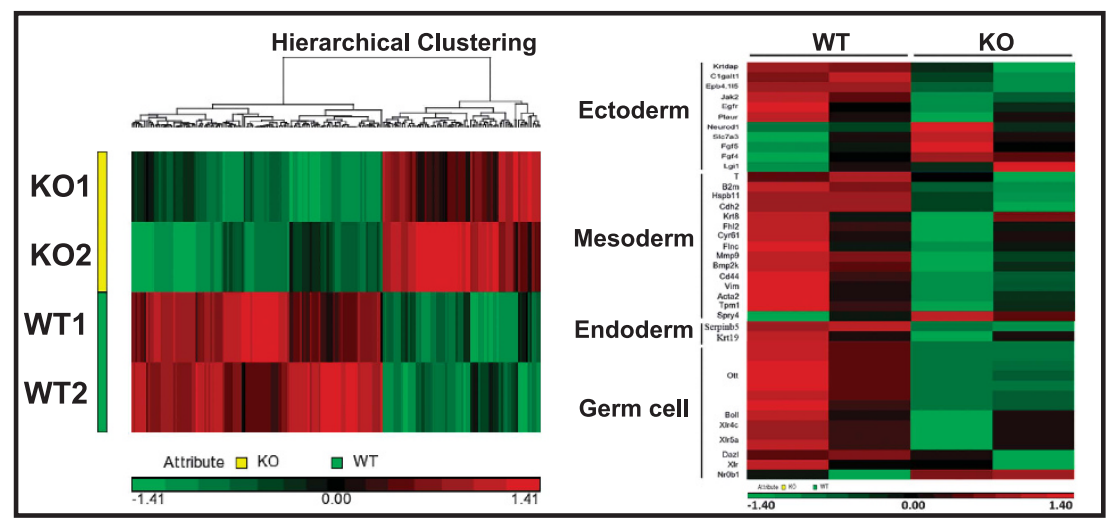

d

Mesoderm/Mesendoderm
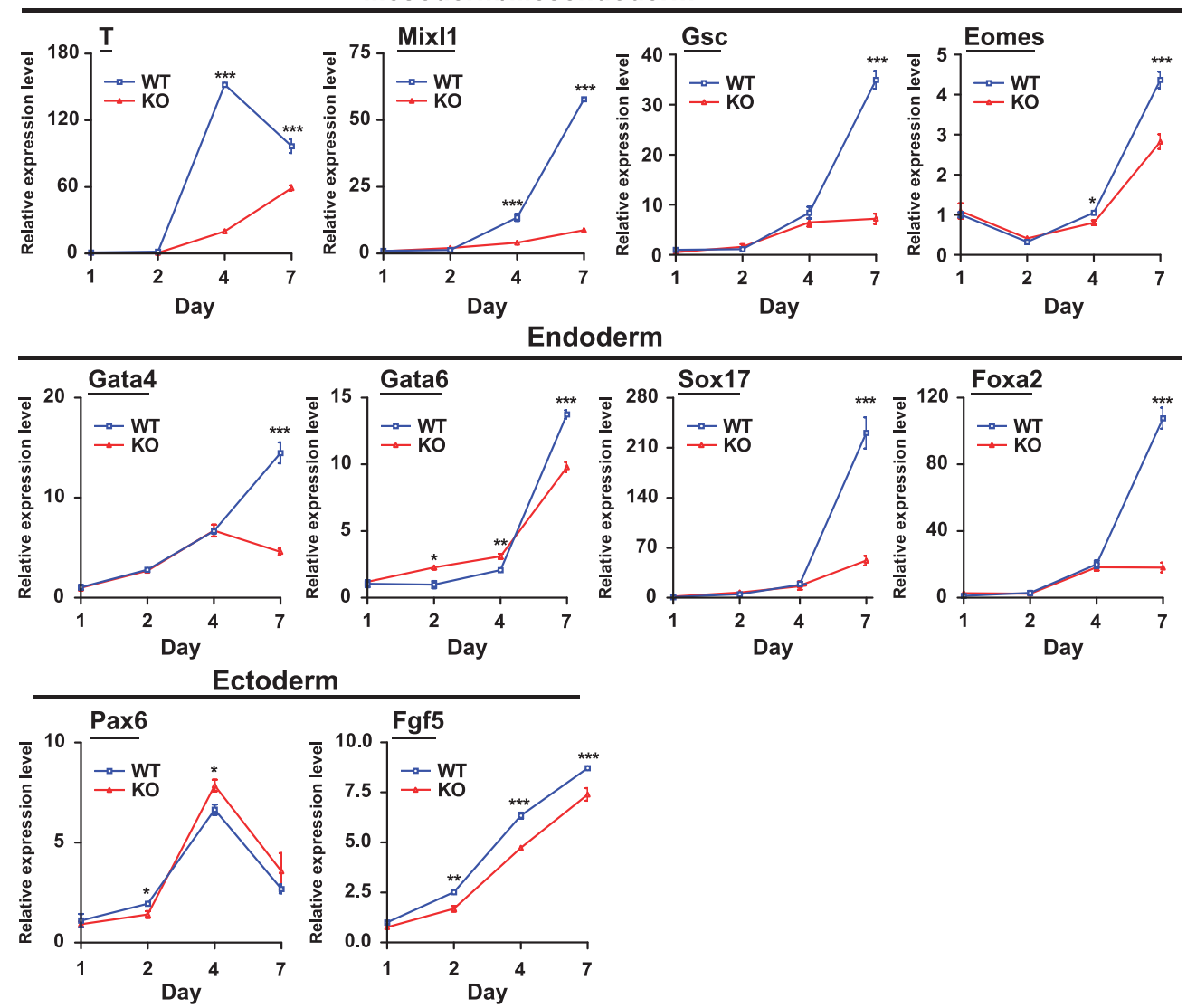
a
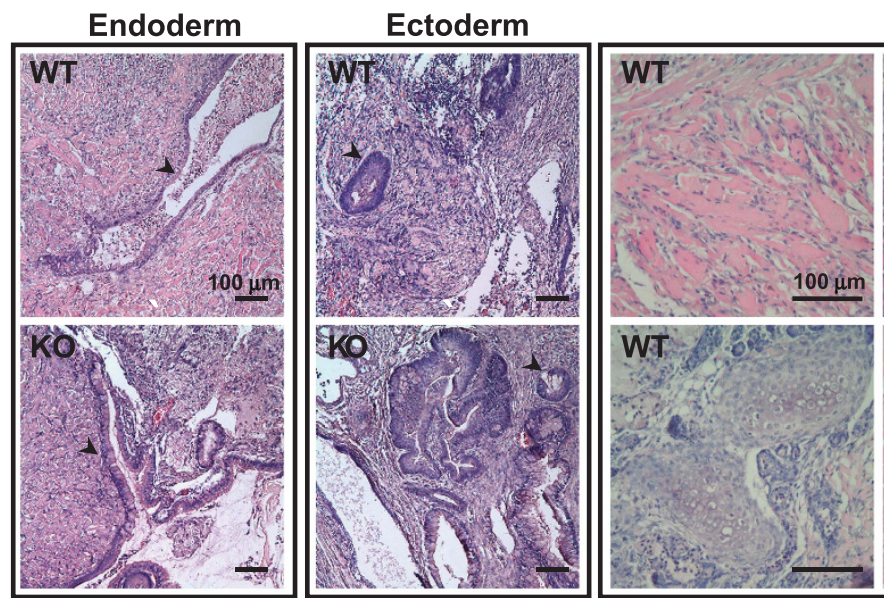

Mesoderm
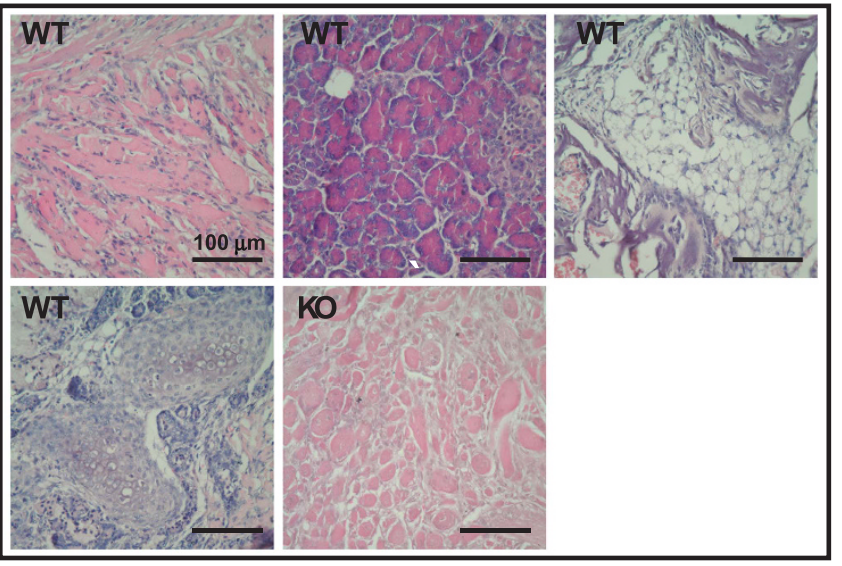

b
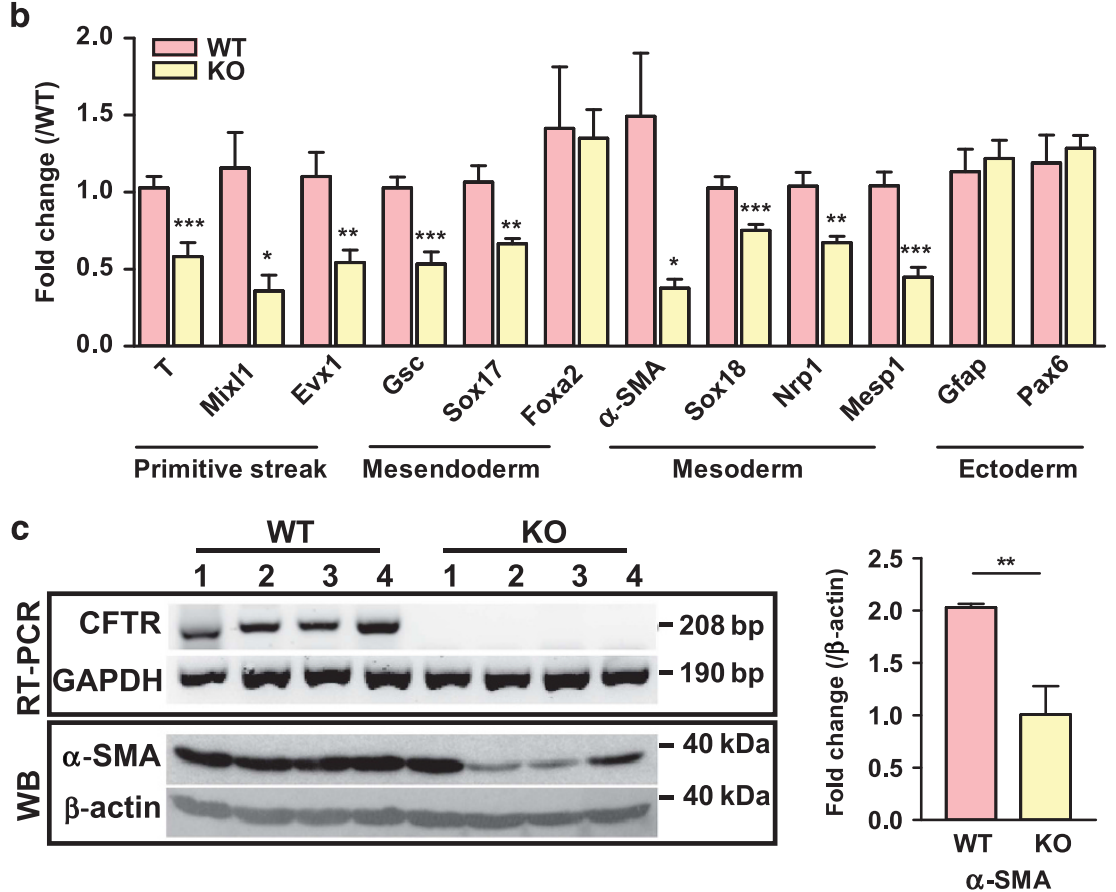

Figure 3 CFTR deficiency impairs mesoderm differentiation in teratomas. (a) Hematoxylin/eosin (H\&E) staining results of WT or KO mESC-formed teratomas; although all three germ layers (ectoderm, mesoderm and endoderm) can be observed in both WT and KO teratomas, more mature tissues derived from mesoderm presented in WT teratomas compared with KO; (b) real-time PCR analysis of three germ layer markers in teratomas formed from WT $(n=4)$ or KO $(n=4) \mathrm{mESCs}$, ${ }^{*} P<0.05$, ${ }^{* \star} P<0.01$ and ${ }^{* * *} P<0.001$; (c) $\alpha$-SMA (mesoderm marker) and CFTR expression in teratomas formed from WT $(n=4)$ or KO $(n=4)$ mESCs, ${ }^{* *} P<0.01$

and KO mESCs. Western blot analysis showed that the total and active $\beta$-catenin in the nuclear extractions in KO mESCs were significantly reduced compared with that in WT (Supplementary Figure S6a). Consistently, the mRNA expression levels of $\beta$-catenin target genes, such as Lef1, c-Jun, Ccnd1, Ccnd2 and Axin2 were significantly decreased in KO mESCs compared with that in WT mESCs (Figure 4a). In addition, whereas the basal level of $\beta$-catenin transcriptional activity was low and no significant difference was observed between WT and KO mESCs, the luciferase activity of $\beta$-catenin was significantly decreased in the KO mESCs when the cells were treated with Wnt3a or CHIR (Supplementary Figure S6b), indicating the effect of CFTR on $\beta$-catenin signaling was more prominent when the pathway was activated. Given that $\beta$-catenin signaling is activated and essential during mesendoderm differentiation in mESCs, we further investigated the regulatory role of CFTR in $\beta$ catenin signaling during $\mathrm{mEB}$ formation. Our western blot results showed that although the expression level of total $\beta$ catenin was similar between WT and KO mEBs, the activated form of $\beta$-catenin, which is destined for nuclear translocation, was significantly decreased in $\mathrm{KO}$ mEBs compared with 
a

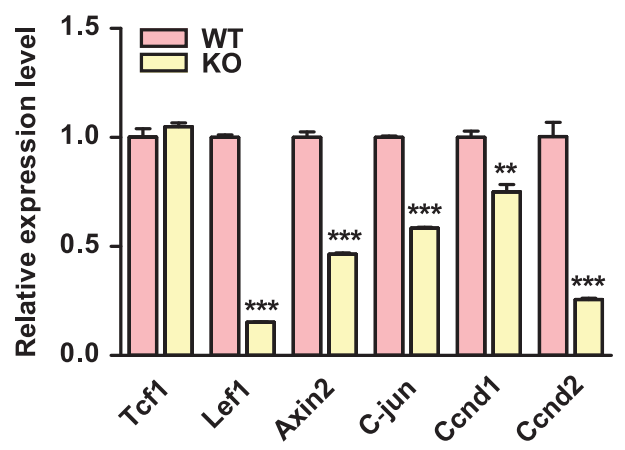

b

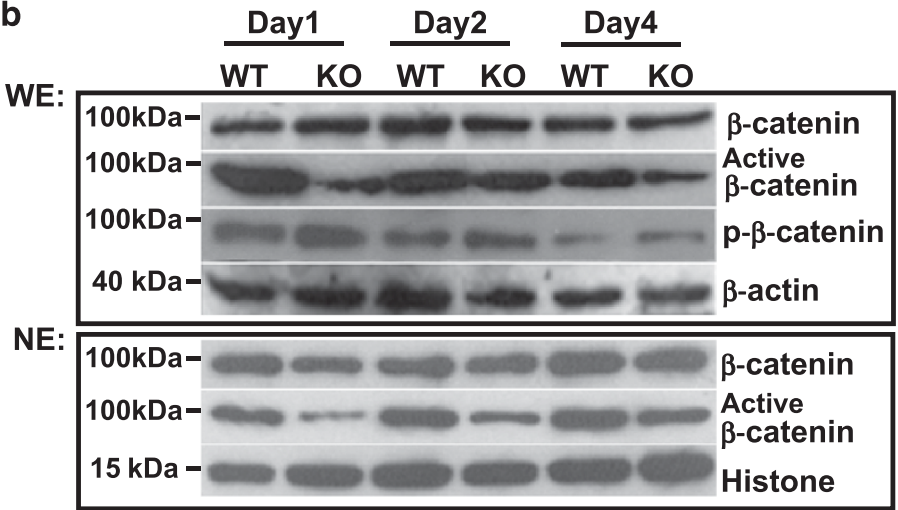

C

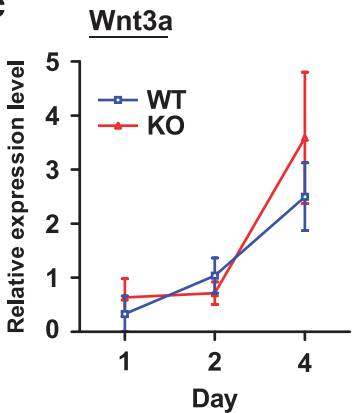

Axin2

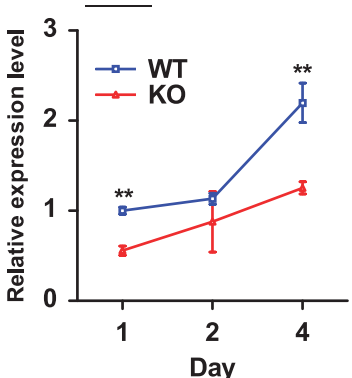

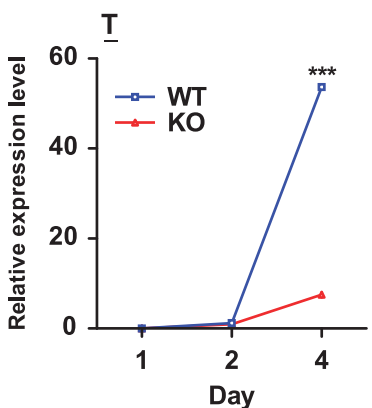

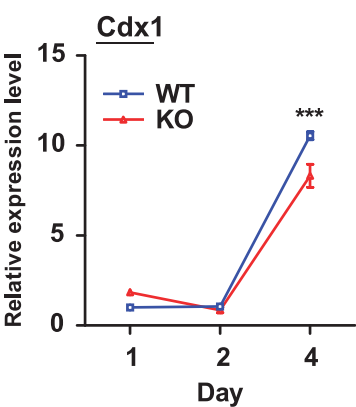

d

$$
\begin{array}{llllllll}
\multicolumn{3}{c}{\text { WT }} & & \multicolumn{3}{c}{\text { KO }} \\
\cline { 5 - 7 } \cline { 5 - 7 } & 2 & 3 & 4 & & 2 & 3 & 4
\end{array}
$$

WE:

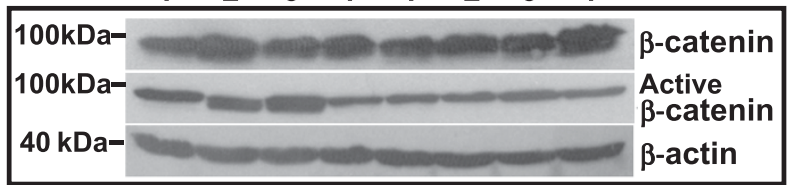

NE:

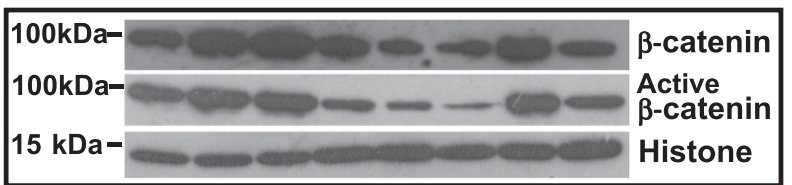

WE

NE

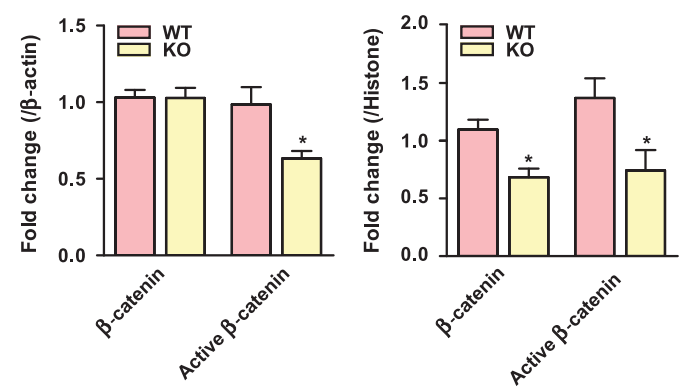

e

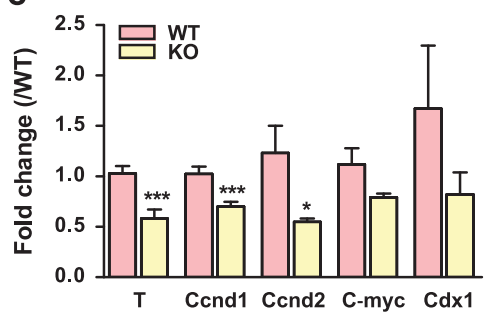

f
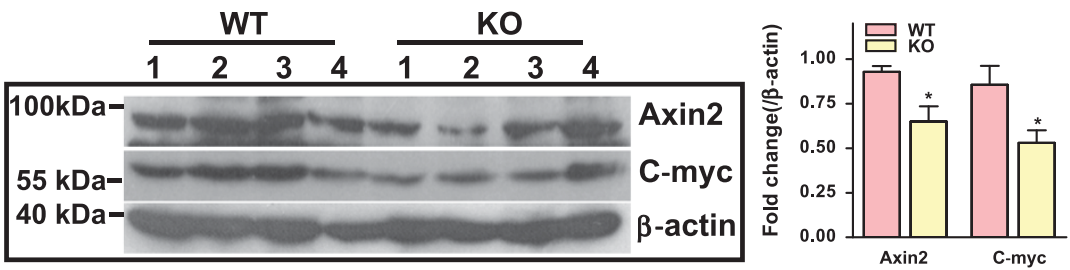

Figure 4 CFTR deficiency downregulates Wnt/ $\beta$-catenin signaling pathway. (a) real-time PCR analysis of Wnt pathway-focused genes in WTand KO mESCs (LIF/CHIR/PD); (b) western blot analysis showing the expression of total $\beta$-catenin, active $\beta$-catenin and $\mathrm{p}$ - $\beta$-catenin in whole extracts (WE) and total $\beta$-catenin and active $\beta$-catenin in nuclear extracts (NE) of WT and KO mEBs induced from mESCs at day 1,2 and 4; (c) real-time PCR analysis of Wnt3a and Wnt pathway targets $A x i n 2, C d x 1$ and $T$ in WTand KO mEBs induced from mESCs at day 1,2 and 4; each dot in the figure represents the mean \pm S.E.M. of triplicates. ${ }^{\star} P<0.05,{ }^{\star \star} P<0.01$ and ${ }^{* \star *} P<0.001$; (d) western blot analysis showing the expression of total $\beta$-catenin and active $\beta$-catenin in WE and NE of WT $(n=4)$ and $\mathrm{KO}(n=4)$ teratomas in 4 weeks, with corresponding statistic summary shown on the right; (e) real-time PCR analysis of Wnt downstream targets T, Ccnd1, Ccnd2, C-myc and Cdx1 in WT and KO teratomas in 4 weeks; (f) western blot analysis of Wnt downstream targets Axin2 and C-myc in WE prepared from WT $(n=4)$ and KO $(n=4)$ teratomas in 4 weeks, with corresponding statistic summary shown in lower panel; (d-f) Each bar in the figure represents the mean \pm S.E.M. of 4 mice from WT and KO groups, separately; ${ }^{*} P<0.05,{ }^{* \star} P<0.01$ and ${ }^{* * *} P<0.001$ 
WT mEBs (Figure 4b). In contrast, the expression level of PS45- $\beta$-catenin, which primes $\beta$-catenin for phosphorylation by GSK3 $\beta$ and subsequent degradation, was significantly increased in KO mEBs (Figure 4b, upper panel). In addition, the nuclear expression of active $\beta$-catenin was dramatically decreased in $\mathrm{KO}$ mEBs (Figure $4 \mathrm{~b}$, lower panel). Using realtime PCR, we then examined the expression of $\beta$-catenin targets that have been implicated in mesendoderm differentiation, and found that the expression levels of Axin2, Cdx1 and $T$ were significantly decreased in $\mathrm{KO} \mathrm{mEBs}$ at day 4 (Figure 4c). The regulatory effect of CFTR on $\beta$-catenin pathway was further validated in teratomas. The expression levels of active $\beta$-catenin were significantly reduced in whole and nuclear extractions of $\mathrm{KO}$ teratomas compared with that of WT teratomas (Figure $4 d$ ). In addition, the expression of Wnt/ $\beta$-catenin pathway targets was also significantly decreased in KO teratomas at both mRNA (Figure 4e) and protein (Figure 4f) levels. Altogether, the results indicate that CFTR deficiency represses $\beta$-catenin signaling pathway during differentiation.

CFTR regulates mESC mesendoderm differentiation via its direct interaction with $\boldsymbol{\beta}$-catenin. As previous proteomics study indicated a potential interaction between CFTR and components of $\mathrm{Wnt} / \beta$-catenin pathway, ${ }^{27}$ we suspected possible protein-protein interaction between CFTR and $\beta$-catenin, where by CFTR stabilizes $\beta$-catenin, defect of which leads to accelerated phosphorylation and premature degradation. To prove this scenario, we first used a protein synthesis inhibitor, cycloheximide $(\mathrm{CHX})$, to block the de novo synthesis of $\beta$-catenin, so that degradation process could be detected. Our results showed that the protein degradation of $\beta$-catenin in KO mESCs was significantly faster than that in WT mESCs (Figure 5a), suggesting that the loss of CFTR results in increased $\beta$-catenin degradation. Next, we checked whether CFTR and $\beta$-catenin were co-localized in undifferentiated and differentiating mEBs. Results of immunofluorescent staining showed that CFTR co-localized with $\beta$-catenin not only at the membrane, but also within the cytoplasm in both undifferentiated and differentiating mEBs (Figure 5b and Supplementary Figure S7a). Then we used co-immunoprecipitation (co-IP) to further test if CFTR physically interacted with $\beta$-catenin in mESCs. As shown in Figure $5 \mathrm{c}$, we found that both CFTR and $\beta$-catenin were detected in the immunoprecipitates of either anti-CFTR or anti- $\beta$-catenin antibody, but not in the immunoprecipitates of IgG control, suggesting that CFTR interacted with $\beta$-catenin in undifferentiated mESCs. The physical interaction of endogenous CFTR and $\beta$-catenin was confirmed in differentiating mEBs at day 4 (Supplementary Figure S7b). These results indicate that CFTR interacts with $\beta$-catenin, affecting $\beta$-catenin phosphorylation and degradation.

We then attempted to rescue the differentiation defect in $\mathrm{KO}$ mEBs with CHIR99021, which inhibits Gsk3 $\beta$ and thus prevents $\beta$-catenin phosphorylation and degradation. ${ }^{18}$ After incubation with CHIR99021 ( $2 \mu \mathrm{m})$ for 4 days, both WT and KO $\mathrm{mEBs}$ showed significantly elevated expression of $\mathrm{Wnt} / \beta$ catenin targets (Axin2, $C d x 1$ ) with no significant difference detected between WT and KO mEBs (Figure 5d). Importantly, the differences in mesendoderm markers ( $T$, Eomes) between
WT and KO mEBs were also completely abolished by CHIR99021 (Figure 5e). To further confirm the involvement of $\beta$-catenin in mediating the effect of CFTR in regulating mESCs differentiation, we overexpressed $\beta$-catenin in $\mathrm{KO}$ mESCs using pT3- $\Delta$ N90 (constitutively active form of $\beta$-catenin) and determined the expression of mesendoderm markers. The result showed that overexpression of $\beta$-catenin completely rescued the repressed mesendoderm markers and $\beta$-catenin targets in $\mathrm{KO}$ mEBs (Figure $5 \mathrm{f}$ ). These results indicate that CFTR regulates mesendoderm differentiation in mESCs via $\beta$-catenin pathway. Moreover, to validate the specific effect of CFTR, we reintroduced human CFTR into KO mESCs and examined whether the suppressed $\beta$-catenin and normal function of differentiation could be restored. Our result showed that although the expression levels of $p$ - $\beta$-catenin were significantly decreased in both WT and KO mEBs with forced expression of CFTR, the level of $p$ - $\beta$-catenin was much higher in $\mathrm{KO}$ mEBs than that in WT mEBs (Supplementary Figure S8c). In addition, overexpression of CFTR partially rescued the decreased $\beta$-catenin activity and mesendoderm differentiation defect exhibited by KO mESCs (Supplementary Figure S8b and d).

Knockdown of CFTR impairs early development in Xenopus laevie via suppression of $\boldsymbol{\beta}$-catenin. To determine the role of CFTR in embryo development, we made use of the Xenopus laevis embryo model, which is a wellestablished model system for the study of embryo development. We performed loss-of-function of CFTR in Xenopus laevis embryos using CFTR antisense morpholino (MO) nucleotide. Injection of $25 \mathrm{ng}$ CFTR MO into both blastomeres of two-cell-stage embryos resulted in an increase in the number of embryos exhibiting gastrulation defect and the number of death. Moreover, those embryos that survived exhibited various abnormal features including eye defects, head malformations and curved body axis (Figure 6a). We counted and divided the embryos at stage 36 into three categories: normal, abnormal, which included all forms of abnormalities, and dead. The quantification results showed that $72.9 .4 \%$ (97 out of 133 embryos) of embryos were dead, $27.1 \%$ (36 out of 133 embryos) showed abnormal phenotype and none (0 out of 133) was normal in CFTR MO group (Figure 6b). In contrast, $4.0 \%$ (7 out of 177 embryos) of embryos were dead, $7.9 \%$ (14 out of 177 embryos) showed abnormal phenotype and $88.1 \%$ (156 out of 177 ) was normal in control group. Importantly, the defects induced by CFTR MO could be partially rescued by injection of human CFTR mRNA into two-cell-stage embryos, suggesting the specificity of CFTR MO (Supplementary Figure S9a). To further investigate the underlying mechanism as to how CFTR regulates embryonic development, the CFTR morphants were harvested at stage 12.5 and examined for dynamic expression of germ layer marker genes, including Xbra, Gsc, Chordin and Vent1 for mesoderm, Sox17 , Foxa2 and Gata6 for endoderm, and Sox2, Sox3 and SoxD for ectoderm by real-time PCR. Consistent with the results from $\mathrm{mESC}$ in vitro differentiation data, the expression of the mesoderm and endoderm marker genes examined was significantly downregulated in CFTR morphants compared with that expressed in control embryos (Figure 6c). These results suggest that 
a
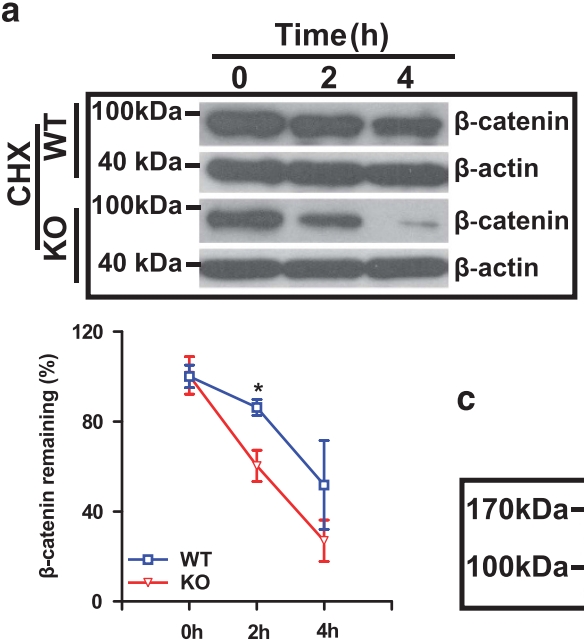

d

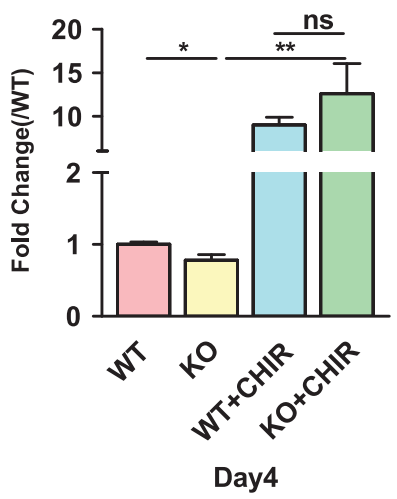

c b

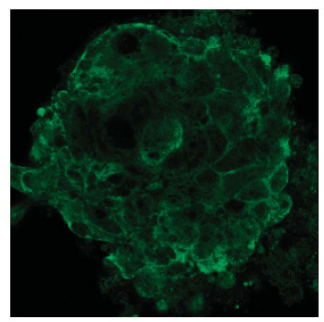

$\beta$-catenin

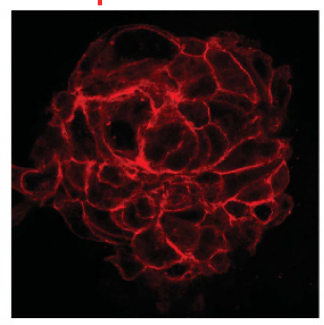

IP

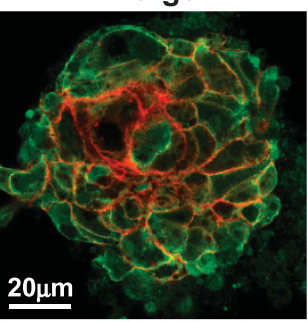

IP

input $\lg \mathrm{G} \beta$-catenin

\begin{tabular}{|l|l|ll|}
\hline $170 \mathrm{kDa}-$ & CFTR & $100 \mathrm{kDa}-$ & $\beta$-catenin \\
$100 \mathrm{kDa}-$ & $\beta$-catenin & $170 \mathrm{kDa}-$ & CFTR \\
\hline
\end{tabular}

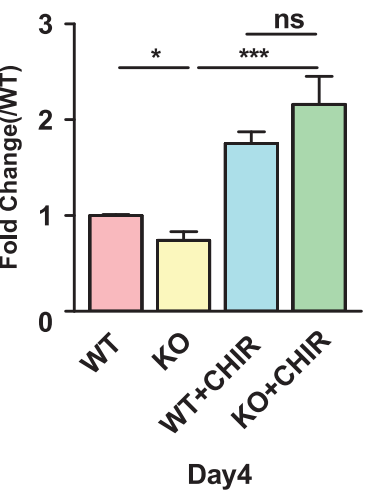

e

$\mathbf{T}$

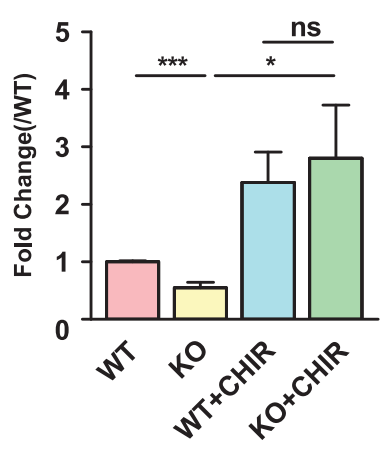

Day4
Eomes

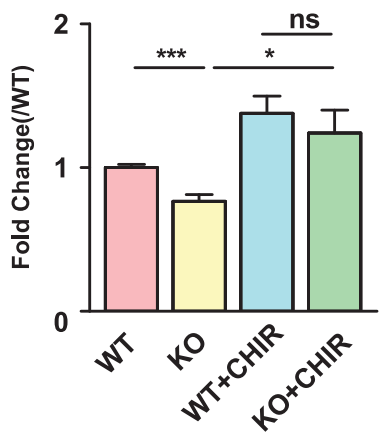

Day4
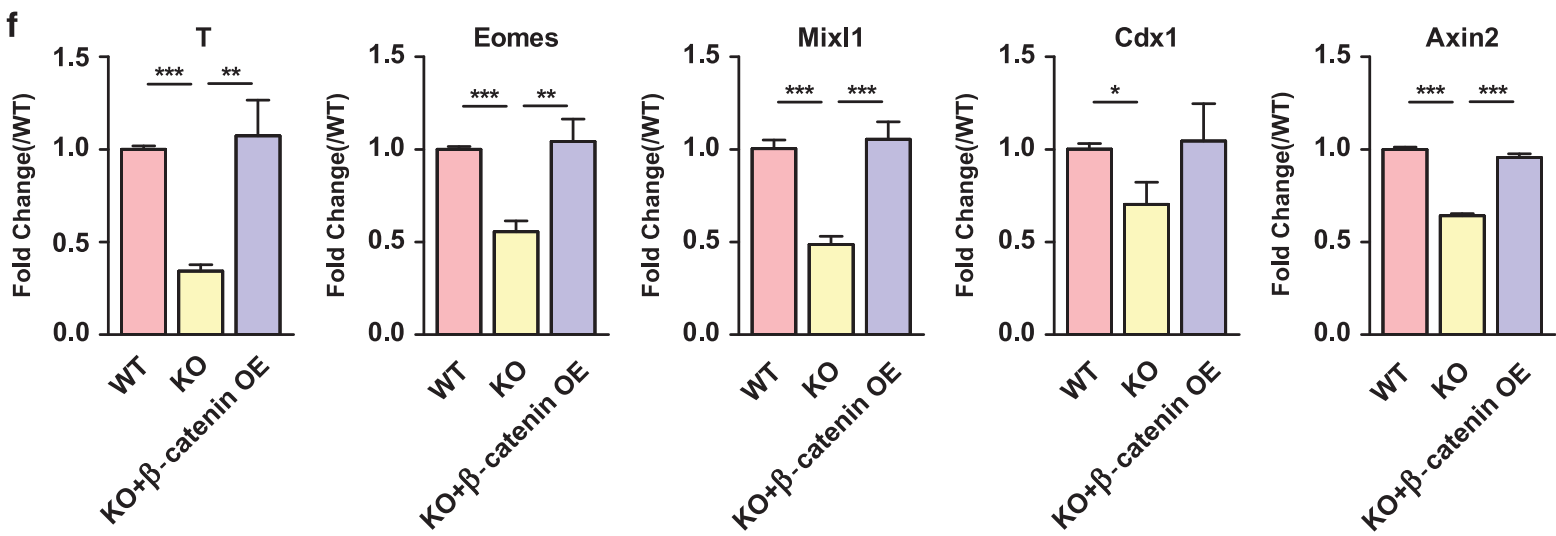

Figure 5 CFTR regulates mESC mesendoderm differentiation via its direct interaction with $\beta$-catenin. (a) In the presence of protein synthesis inhibitor cycloheximide $(100 \mu \mathrm{g} / \mathrm{ml} \mathrm{CHX})$, protein degradation of $\beta$-catenin in KO mESCs is significantly faster than that in WT mESCs $\left({ }^{*} P<0.05, n=3\right)$; (b) fluorescent staining of CFTR and $\beta$-catenin showing co-localization of CFTR (green) and $\beta$-catenin (red) in mEBs; (c) Co-IP of endogenous CFTR and $\beta$-catenin; non-denatured whole-cell extracts prepared from mESCs were immunoprecipitated with anti-CFTR, anti- $\beta$-catenin or control IgG antibody; the immune complexes and the input (10\% of the whole-cell extracts used in the immunoprecipitation step) were analyzed by immunoblotting with CFTR and $\beta$-catenin antibody; (d) real-time PCR analysis showing mRNA expression of Wnt targets (Axin2, Cdx1) in WT and KO mEBs was dramatically increased when treated with CHIR $(2 \mu \mathrm{M})$ for 4 days; (e) real-time PCR analysis showing the differential mRNA expression of mesendoderm markers (T, Eomes) in WTand KO mEBs was rescued by CHIR $(2 \mu \mathrm{M})$ treatment; (f) real-time PCR analysis showing ectopic expression of constitute $\beta$-catenin rescued the defected mesendoderm differentiation and $\beta$-catenin activation in $\mathrm{KO} \mathrm{mEBs}$

CFTR is crucial for early embryonic development in the Xenopus laevis in vivo.

During Xenopus development, i-catenin signaling is critical for mesoderm-endoderm induction. ${ }^{30,31}$ To further investigate whether CFTR regulates $\beta$-catenin signaling during Xenopus embryo development, we injected TOP-flash luciferase Wnt/ $\beta$ catenin reporter in the two-cell stage of control and CFTR MO embryos and determined the reporter activity at stage 12.5. 

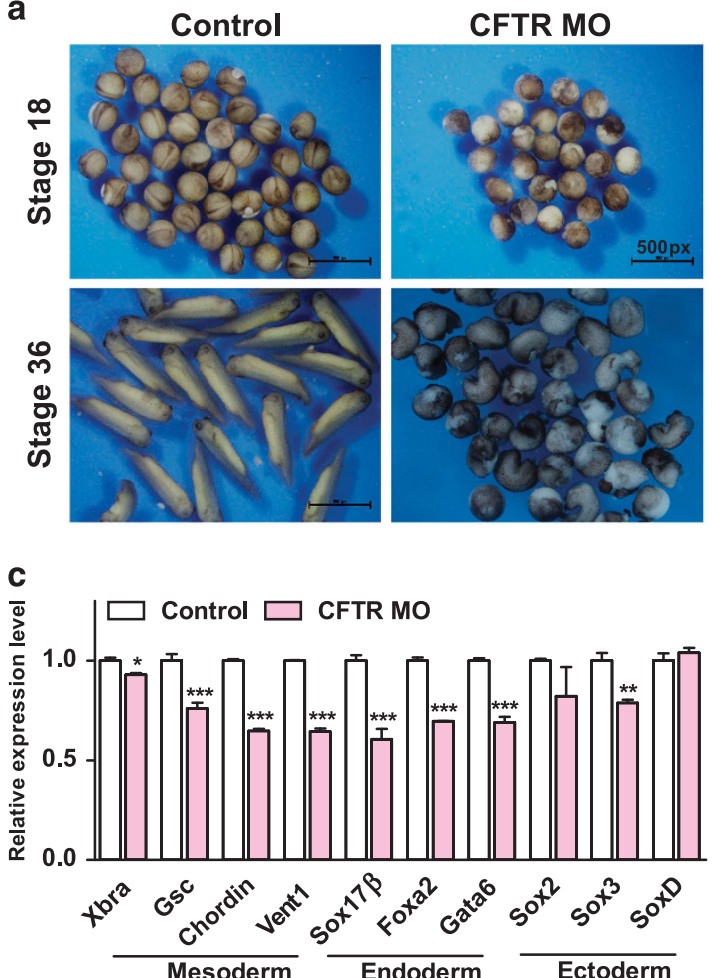

f

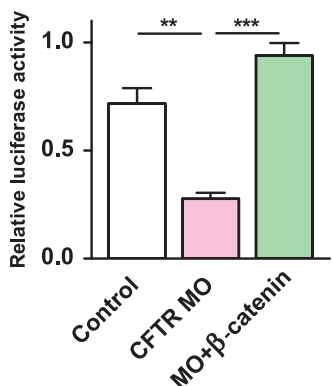

CFTR MO d
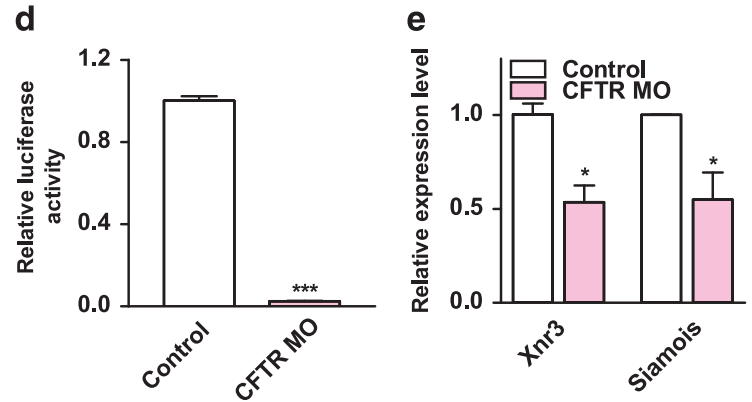

h

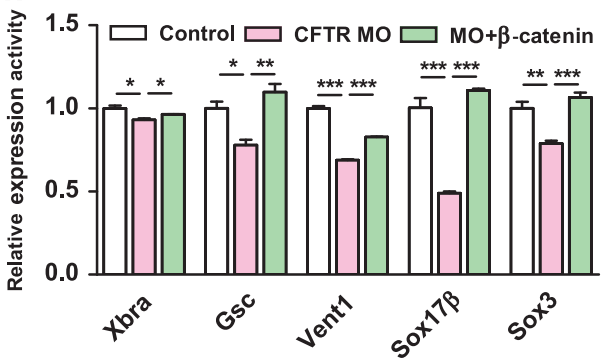

Figure 6 Knockdown of CFTR in Xenopus laevie embryos impairs early development and $\beta$-catenin signaling and its rescue. (a)The phenotypes of embryos injected with $25 \mathrm{ng}$ of CFTR MOs; (b) quantification of aberrant phenotypes induced by CFTR MO; the phenotypes are divided into 3 categories, normal, abnormal and dead; the abnormal embryos showed short and curved body; (c) real-time PCR analysis of three germ layer markers: mesoderm (Xbra, Gsc, Chordin, Vent1), endoderm (Sox 17 $\beta$, Foxa2, Gata6) and ectoderm (Sox2, Sox3, SoxD) in total mRNA of whole embryos at stage 12.5 injected Control MO (Ctrl) or CFTR MO (25 ng); (d) luciferase analysis showing $\beta$-catenin activity in whole embryos at stage 12.5 injected Control MO or CFTR MO (25 ng); (e) real-time PCR analysis of Wnt pathway targets Xnr3 and Siamois in total mRNA of whole embryos at stage 12.5 injected Control MO and CFTR MO $(25 \mathrm{ng})$; (f-h) rescue of CFTR MO-induced defects in Xenopus development by overexpressing $\beta$-catenin; luciferase analysis showing $\beta$-catenin activation (f), real-time PCR analysis of Wnt pathway targets, Xnr3 and Siamois, in whole embryos at stage 12.5 (g), real-time PCR analysis of three germ layer markers (h); each bar in the figure represents the mean \pm S.E.M. of triplicates; ${ }^{\star} P<0.05,{ }^{\star \star} P<0.01$ and ${ }^{\star \star \star} P<0.001$

As shown in Figure 6d, the relative luciferase activity reflecting the outputs of Wnt/ $\beta$-catenin signaling was dramatically reduced in CFTR morphants. Moreover, the expression levels of Wnt/ $\beta$-catenin signaling targets including $X n r 3$ and Siamois were also significantly decreased in CFTR morphants compared with that in control embryos (Figure 6e). We then tried to rescue the defective phenotype in CFTR MO embryos by restoring active $\beta$-catenin. To do this, we injected $\beta$-catenin mRNA (100 pg) into CFTR MO embryos at two-cell stage and examined the development of embryos at stage 12.5 . Our results showed that with the concentration that could completely restore the transcriptional activity and expression of downstream targets (Figure $6 \mathrm{f}$ and $\mathrm{g}$ ), ectopic expression of $\beta$-catenin in CFTR MO embryos significantly reversed the downregulation of the germ layer markers including mesoderm (Xbra, Gsc, Vent1), endoderm (Sox17 $\beta$ ) and ectoderm (Sox3) in CFTR MO embryos (Figure 6h). Finally, we tried to confirm the specific effect of CFTR MO by overexpressing human CFTR mRNA in CFTR MO embryos. The result showed that CFTR mRNA expression completely reversed the inhibitory effect of CFTR MO on the expression of mesendoderm markers (Supplementary Figure S9b). Taken together, 
results from Xenopus laevis embryos confirm a critical role of CFTR in early embryonic development.

\section{Discussion}

The present study has revealed a previously unrecognized role of CFTR in ESC differentiation, which is fundamentally important for embryonic development. Our microarray data showing downregulation of multiple mesoderm and endoderm markers in $\mathrm{KO}$ mEBs at day 5, is consistent with the notion that CFTR deficiency impairs the mesendoderm commitment which corresponds to primitive streak formation stage during embryonic development in vivo (Figure 2c). Specifically, we have shown that Brachyury ( $T$ ) and Mix/1, which are first expressed in the early primitive streak-stage embryo, ${ }^{32,33}$ are dramatically downregulated in $\mathrm{KO}$ mEBs at day 4. Following that, genes reflective of mesoderm and endoderm differentiation (Gsc, Eomes, Gata4, Gata6, Sox17, Foxa2) are prominently downregulated at day 7 (Figure 2d). These results clearly demonstrate that CFTR is critical for the early mesendoderm differentiation in mESCs. Although the role of CFTR in the late differentiation stage for mesendoderm is still not understood, an interesting finding from our teratomaformation assay is that only mesoderm but not endoderm differentiation is dramatically disrupted in the CFTR KO teratomas (Figure $3 a-c$ ), indicating the presence of alternative regulatory pathways in the late endoderm differentiation of mESCs.

Wnt/ $\beta$-catenin signaling pathway has a central role in controlling lineage specification during early embryogenesis. ${ }^{13,14}$ Consistent with the previous study, our present study shows the expression levels of active $\beta$-catenin and $\beta$-catenin target genes in $\mathrm{KO} \mathrm{mEBs}$ are significantly downregulated compared with WT mEBs (Figure $4 a-c$ ). Moreover, $\beta$-catenin signaling is also dramatically suppressed in $\mathrm{KO}$ mESC-injected teratomas (Figure $4 \mathrm{~d}-\mathrm{f}$ ). These results indicate that CFTR regulates $\beta$-catenin-mediated signaling pathway, which is of paramount importance for mesendoderm differentiation and embryonic development. Of particular interest, for the first time, we have shown that CFTR physically interacts with $\beta$-catenin in mESCs and mEBs, and they are co-localized in both membrane and cytoplasm (Figure $5 b, c$, Supplementary Figure S7a and b). Given that stabilization of $\beta$-catenin is pivotal for its subsequent nuclear translocation and activation, we propose that the direct interaction between CFTR and $\beta$-catenin prevents $\beta$-catenin phosphorylation and degradation, defect of which results in augmented phosphorylation and premature degradation as observed (Figure 5a). This notion is supported by the results using GSK3 $\beta$ inhibitor, which prevents $\beta$-catenin degradation, reverses the suppressive effect of CFTR deficiency on mesendoderm differentiation and $\beta$-catenin target expression in $\mathrm{KO}$ mESCs (Figure $5 d$ and e). Thus, our study has revealed a novel regulatory mechanism where by $\beta$-catenin activity is dictated by its interaction with CFTR, which is critical for the early mesendoderm commitment in mESCs.

The role of CFTR in $\beta$-catenin signaling and early development is further demonstrated in the present study in the Xenopus laevis in vivo. We have shown that knockdown of CFTR dramatically impairs the embryo development of
Xenopus at very early stage, and represses the expression of multiple mesoderm and endoderm genes at stage 12.5 (Figure 6a-c). The phenotype shown in the CFTR MO-treated embryos is striking, probably due to the high demand of $\beta$-catenin activity in Xenopus development. ${ }^{34-36}$ The present study has shown that the loss of CFTR suppresses $\beta$-catenin transcriptional activity and Wnt/ $\beta$-catenin downstream targets that are directly involved in the mesendoderm induction, such as $X n r 3$ and Siamois (Figure $6 \mathrm{~d}$ and e). These results indicate that CFTR is critical for the mesendoderm differentiation during embryo development via regulation on $\beta$-catenin pathway in vivo. It should be noted that some of the differentiation markers could only be partially rescued by $\beta$-catenin overexpression in CFTR MO embryos (Figure 6h), indicating that CFTR may regulate other key signaling pathways involved in Xenopus early development. Indeed, previous studies have shown that CFTR can regulate $\mathrm{TGF} \beta^{37}$ and $E R K^{38}$ pathways, which are also known to be involved in Xenopus embryonic development.

Of particular interest, our preliminary results obtained from $\triangle \mathrm{F} 58^{-/-} \mathrm{mEBs}$ also showed impaired mesoderm differentiation (Supplementary Figure S10f) and increased expression of p- $\beta$-catenin (Supplementary Figure S10h) compared with the WT mEBs. In addition, the expression of total and active $\beta$-catenin in the nuclear fraction of $\triangle F 508$ mESCs was dramatically decreased (Supplementary Figure S10g). These results suggest that many of the clinical manifestations observed in CF patients, with $\Delta \mathrm{F} 508$ as the major mutation, may actually stem from developmental defects due to defective $\beta$-catenin signaling as demonstrated in the present studies. Interestingly, two recent studies clearly showed that the $\triangle$ F508-CFTR mutation impaired the differentiation of bone marrow stromal cells into osteoblasts and the activity of osteoblasts. Consistent with our results, these studies also found increased $\beta$-catenin phosphorylation, reduced osteoblast $\beta$-catenin expression and altered expression of $\mathrm{Wnt} / \beta$-catenin target genes in $\Delta \mathrm{F} 508$ mutant osteoblasts. ${ }^{39,40}$ Given that bone is of mesoderm origin, it is plausible that the defected mesoderm differentiation reported in our study contributes to the pathological condition of bone in $\Delta \mathrm{F} 508$ mice. Collectively, in view of the importance of $W n t / \beta$-catenin pathway in embryonic development, the presently demonstrated critical role of CFTR in regulating $\beta$-catenin signaling provides novel insights into the molecular mechanism underlying embryonic development.

\section{Materials and Methods}

Mouse embryos and establishment of mESCs. Mice were obtained from the laboratory animal service center (LASEC) of the Chinese University of Hong Kong (CUHK, Hong Kong, China). CFTR ${ }^{\text {tmiUnc }}$ (CFTR KO) mice, with a neomycin selection cassette inserted into exon 10 at sequences corresponding to codon 489 of the encoded CFTR protein, ${ }^{41}$ were obtained from the Jackson Laboratory, Bar Harbor, ME, USA and maintained in LASEC of CUHK. Wild-type and CFTR $^{-1-}$ knock-out mouse embryos were obtained by crossing CFTR ${ }^{\text {tmiUnc }}$ $(+/-)$ female and male mice. E3.5 blastocysts were flushed out from the uterus and cultured on mitomycin C-treated MEF feeder in a 96-well plate with N2B27 medium with 2i (PD0325901, $0.4 \mathrm{uM}$; CHIR99021, $3 \mu \mathrm{M}$ ) and LIF (1000 U/mI). The ICM outgrowth was treated with $0.05 \%$ Trypsin (Gibco Life Technologies, Carlsbad, CA, USA) and passaged on a 24-well plate until stable mESC lines were obtained. The mESCs were maintained on feeders under the normal mESC medium (DMEM supplemented with $15 \%$ FBS, $0.1 \mathrm{~mm}$ non-essential amino acids, $0.1 \mathrm{~mm}$ 
2-mercaptoethanol, $2 \mathrm{mM}$ Glutamine, $100 \mathrm{U} / \mathrm{ml}$ penicillin/streptomycin and $1000 \mathrm{U} / \mathrm{ml} \mathrm{LIF)}$. To obtain feeder-free mESCs, the mESCs were grown on $0.1 \%$ Gelatin-coated dish in 2i (PD0325901, $1 \mu \mathrm{M}$ and CHIR99021, $3 \mu \mathrm{M}$ )+LIF $(1000 \mathrm{U} / \mathrm{ml})$. Cells were maintained in $37^{\circ} \mathrm{C}$ and $5 \% \mathrm{CO}_{2}$ incubator.

Xenopus laevis embryo manipulation and microinjection. Female frog ovulation was stimulated by injection with $500 \mathrm{IU}$ of human chorion gonadotropin (Sigma-Aldrich, St Louis, MO, USA) into the dorsal lymph sac. In vitro fertilization was performed by mixing male testis homogenates and eggs. After fertilization, embryos were dejellied with $2 \%$ cysteine solution, $\mathrm{pH} 8$ and cultured in $0.2 \times$ MMR $\left(20 \mathrm{~mm} \mathrm{NaCl}, 0.2 \mathrm{~mm} \mathrm{KCl}, 0.2 \mathrm{~mm} \mathrm{MgSO}_{4}, 0.4 \mathrm{~mm} \mathrm{CaCl}\right.$, $0.02 \mathrm{~mm}$ EDTA, $1 \mathrm{~mm}$ HEPES, pH 7.8) as previously described. ${ }^{42}$ Embryos were staged according to Nieuwkoop and Faber. ${ }^{43}$ 2-cell stage Xenopus laevis embryos were used for microinjection. Prior to each injection, the injection needle was washed twice with DEPC- $\mathrm{H}_{2} \mathrm{O}$ to remove RNase contamination. The procedure was carried out in $4 \%$ ficoll 400 dissolved in $1 \times$ MMR, which could collapse the vitelline envelop and minimize cytoplasm leakage during injection. Microinjection is performed at about $22^{\circ} \mathrm{C}$. After injection, culture the injected embryos in $0.2 \times$ MBS culture medium at $16^{\circ} \mathrm{C}$ overnight and then transfer to $22{ }^{\circ} \mathrm{C}$.

EB formation. mESCs were trypsinized, re-suspended in EB medium (DMEM supplemented with $10 \%$ FBS, $5 \%$ KSR, $0.1 \mathrm{~mm}$ non-essential amino acids, $2 \mathrm{~mm}$ Glutamine, $100 \mathrm{U} / \mathrm{ml}$ penicillin/streptomycin) at a cell density of $1.5-2 \times 10^{5}$ cells $/ \mathrm{ml}$ and plated on a non-adherent petri dish to initiate EB formation.

mESC Transfection. For $\beta$-catenin and CFTR overexpression in CFTR KO mESCs, pT3-N90- $\beta$-catenin (Addgene Plasmid \#31785) was purchased from Addgene (Cambridge, MA, USA). pT3-N90- $\beta$-catenin is a $\beta$-catenin mutant construct with NH2-terminal deletion of 90 amino acids. pcDNA3.1-CFTR vector was used to overexpress CFTR. The empty vector or pEF-GFP was used as a negative control. Generally, cells were seeded in 6-well culture plate at $1 \times 10^{5}$ cells per well in full medium. After incubation overnight, cell medium was changed to PSfree medium. In all, $2.5 \mu \mathrm{g}$ vector and $5 \mu \mathrm{l}$ lipofectamine 2000 were mixed in $500 \mu \mathrm{l}$ opti-MEMI (for each well) and added to the medium. Fresh full medium was changed after $24 \mathrm{~h}$ incubation. Cells were then trypsinized for further analysis. For the $\beta$-catenin/TCF reporter assay, mESCs were plated on 24-well plates without feeder and transfected by Lipofectamine 2000 (Life Technologies, Carlsbad, CA, USA) with $250 \mathrm{ng}$ of the $\beta$-catenin/TCF reporter constructs (Millipore, Billerica, MA, USA) together with $2.5 \mathrm{ng}$ of pRL-CMV (Renilla luciferase, Promega, Madison, WI, USA) as internal standard for transfection efficiency.

RNA extraction and qRT-PCR. Total RNA of cells was extracted using TRIzol Reagent according to the manufacturer's instruction. The RNA concentration was measured by NanoVue UV/Vis Spectrophotometer. The isolated total RNA was dissolved in DEPC-treated $\mathrm{H}_{2} \mathrm{O}$. In all, 1-5 $\mu \mathrm{g}$ total RNA was applied on reverse transcription reaction using molony murine leukemia virus reverse transcriptase. $1 \mu \mathrm{l} \mathrm{CDNA}$ was mixed with $1 \mu \mathrm{l}$ of $2.5 \mu \mathrm{m}$ forward primer, $1 \mu \mathrm{l}$ of $2.5 \mu \mathrm{m}$ reverse primer, $5 \mu$ l Bio SYBR Green Master mix (Takara, Kusatsu, Shiga, Japan). PCR was performed on Applied Biosystem 7500 fast real-time PCR system (Thermo Fisher Scientific, Waltham, MA, USA). Specific primers were purchased from Life technologies and listed in Supplementary Table S1. To calculate the relative transcriptional expression, the $\mathrm{Ct}$ values of interested genes were normalized by average $\mathrm{Ct}$ values of gapdh as $\Delta \mathrm{Ct}$, The relative transcriptional expression of interested genes was indicated with $2^{(--\Delta \Delta C t)}$.

Protein extraction and western blot. Cells were collected and washed once using PBS. RIPA buffer ( $150 \mathrm{~mm} \mathrm{NaCl}, 50 \mathrm{~mm}$ Tris-Cl, 1\% NP-40, 0.5\% DOC, $0.1 \%$ SDS, 1:100 PMSF and 1:100 PImix) was used to lyse cells for $30 \mathrm{~min}$ at $4{ }^{\circ} \mathrm{C}$. Supernatant was collected as total protein after centrifugation at 12000 r.p.m. for $30 \mathrm{~min}$. For nuclear protein extraction, $50 \mu \mathrm{l}$ (for $60 \mathrm{~mm}$ dish) low-salt buffer (10 mm HEPES, $10 \mathrm{~mm} \mathrm{KCl,} 1 \mathrm{~mm}$ EDTA, 1 mm EGTA, 0.2\% NP-40, 10\% Glycerol) was added to cells. After vortex for $1 \mathrm{~min}$, the microtubes were incubated on ice for $5 \mathrm{~min}$ followed by centrifuging for $2 \mathrm{~min}$ in $4^{\circ} \mathrm{C}$ at 13000 r.p.m. The supernatant was collected carefully as cytoplasmic fraction and stored in $-80^{\circ} \mathrm{C}$. The nuclear pellet was re-suspended with 25-30 $\mu$ l high-salt buffer (20 mM HEPES, $420 \mathrm{~mm}$ $\mathrm{NaCl}, 10 \mathrm{~mm} \mathrm{KCl}, 1 \mathrm{~mm}$ EDTA, $1 \mathrm{~mm}$ EGTA, 20\% Glycerol) and rotated for $30 \mathrm{~min}$ in $4{ }^{\circ} \mathrm{C}$. The nuclear membrane fragments were removed by centrifuging at $13000 \mathrm{r.p}$. $\mathrm{m}$. for $10 \mathrm{~min}$ in $4^{\circ} \mathrm{C}$. The supernatant was collected carefully as nuclear fraction and stored in $-80^{\circ} \mathrm{C}$. Equal amounts of protein were separated by SDS-PAGE and detected for target proteins. The protein bands were visualized by the enhanced chemo-luminescence assay (GE Healthcare, Buckinghamshire, UK) following manufacturer's instructions and scanned by densitometer. Antibodies used are CFTR (1/500, Almone Lab, Jerusalem, Israel, ACL-006), $\beta$-catenin (1/1000, CST, cst9562), non-phospho (Active) $\beta$-catenin (1/1000, CST, Boston, MA, USA, cst8814), phospho- $\beta$-catenin (1/1000, CST, cst9561), axin2 (1/1000, Abcam, Cambridge, MA, USA, ab32197), c-myc (1/1000, CST, cst5605), oct3/4 (1/1000, Santa Cruz, CA, USA, sc9081), nanog (1/1000, Abcam, ab80892), sox2 (1/1000, CST, cst2748), $\beta$-actin (1/2000, Sigma, St. Louis, MO, USA, A1978), Histone1 (1/1000, Santa Cruz, sc8030).

Co-immunoprecipitation. Three groups of cells (grow on $100 \mathrm{~mm}$ dish) were included in co-IP experiment: input group, antibody group and IgG group. The medium of cells were discarded and cells were rinsed in $1 \times$ PBS twice to remove extra medium. Protein interactions were then fixed using $200 \mu \mathrm{g} / \mathrm{ml}$ DTSSP solution for $20 \mathrm{~min}$ at room temperature. The cross link reaction was terminated by $50 \mathrm{~mm}$ Glycine in PBS for 5 min. For antibody and IgG group, cells were lysed with $1 \mathrm{ml}$ of ice-cold IP lysis buffer (50 mm HEPES, $420 \mathrm{~mm} \mathrm{KCl,} \mathrm{0.1 \%} \mathrm{NP-40,} 1$ mm EDTA) for $30 \mathrm{~min}$ to $1 \mathrm{~h}$ on ice. For input group, $100 \mu \mathrm{l}$ of IP lysis buffer was added to cells and lysed for the same time. Supernatants of all groups were collected as cell lysates after centrifugation at 12000 r.p.m. for $15-30 \mathrm{~min}$ in $4^{\circ} \mathrm{C}$. Lysates of input was store at $-80^{\circ} \mathrm{C}$. Lysates of the other two groups were incubated with $5 \mu \mathrm{g}$ anti-CFTR or anti- $\beta$-catenin antibody or normal Rabbit IgG together with Protein $A / G$ beads on rotator overnight in $4{ }^{\circ} \mathrm{C}$. The beads were harvested by centrifugation in the following day and washed 5 times using PBS supplemented with protease inhibitor. $1 \times$ SDS loading buffer was added to beads and incubated for $30 \mathrm{~min}$ at room temperature. The co-IP results were further analyzed by western blotting.

Microarray analysis. Total RNA of WT and CFTR KO mEBs at day 5 was extracted using Trizol (Life Technologies, Carlsbad, CA, USA) according to the instructions. In all, $10 \mu \mathrm{g}$ total RNA was aliquoted and digested with DNAase (New England Biolab, MA, USA) at $37^{\circ} \mathrm{C}$ for 45 min to remove DNA contamination, and then the RNA samples were purified with RNeasy mini-kit (Qiagen, Valencia, CA, USA) according to the instructions. Subsequently, the purified RNAs were reversely transcribed, labeled and hybridized to Affymetrix mouse exon 1.0 ST Array (Santa Clara, CA, USA). All the microarray processes were conducted according to the manufacturer's instructions. Finally, the array data were analyzed using the software Partek and Genespring (Agilent Technology, Santa Clara, CA, USA). Differentially expressed genes were identified on the basis of statistically significant differences in transcript-level signal intensity and a fold change of 1.5 .

Immunofluorescent staining. Cells were grown on $10 \mathrm{~mm}$ glass coverslips pre-coated with $0.2 \%$ gelatin (Sigma-Aldrich, St Louis, MO, USA). After incubation for 3-4 days, cells were fixed in fresh 4\% PFA. The coverslips were air-dried in chemical hood for 5 min and washed with PBS for 5 min twice. Cells were then blocked in $1 \% \mathrm{BSA}$ for $1 \mathrm{~h}$ at room temperature, followed by incubation with primary antibody overnight in $4^{\circ} \mathrm{C}$ refrigerator (CFTR, $1 / 100, A C L-006 ; \beta$-catenin, $1 / 100$, cst2677; Oct4, 1/100, sc9081). In the following day, coverslips were washed with PBS for 3 min twice. Then secondary antibodies were applied on cells for $1 \mathrm{~h}$ at room temperature. After PBS washing, the nucleus was stained with Hoechst 33342. Negative control group that omitted primary antibody was included in this study. The cells were visualized under fluorescent or confocal microscopy.

Intracellular $\mathrm{Cl}^{-}$measurement. Cells grown on $25 \mathrm{~mm}$ diameter glass coverslips were washed 3 times with a Margo solution containing (in $\mathrm{mm}$ ): $130 \mathrm{NaCl}$, $5 \mathrm{KCl}, 2.5 \mathrm{CaCl}_{2}, 1 \mathrm{MgCl}_{2}, 20$ Hepes, 10 glucose, pH 7.4, which were then loaded with $10 \mathrm{~mm}$ MQAE for $30 \mathrm{~min}$ at $37^{\circ} \mathrm{C}$ incubator. After that, the cells were washed twice and kept in a MQAE-free Margo solution for another $30 \mathrm{~min}$ at room temperature in dark. To obtain the maximum fluorescence ( $\mathrm{Fmax}$ ), a $\mathrm{Cl}^{-}$-free Margo solution ( $130 \mathrm{~mm} \mathrm{Na}$ gluconate, $5 \mathrm{~mm} \mathrm{~K}$ gluconate, $20 \mathrm{~mm} \mathrm{Ca}$ gluconate, $1 \mathrm{~mm}$ $\mathrm{MgSO}_{4}, 20 \mathrm{~mm}$ HEPES, $10 \mathrm{~mm}$ glucose, $\mathrm{pH}$ 7.4) with $10 \mu \mathrm{m}$ nigericin and $10 \mu \mathrm{m}$ tributyltin was used. For the minimum fluorescence (Fmin), $150 \mathrm{~mm} \mathrm{KSCN}$ buffered with $10 \mathrm{~mm}$ HEPES ( $\mathrm{pH} 7.4$ ) and $5 \mu \mathrm{m}$ valinomycin was used. To start the experiment, coverslips were transferred to a mini-chamber and maintained in Margo solution. Drugs were added after the baseline was stable. Measurement was made on an epifluorescence microscope (Nikon Eclipse Ti, Konan, Minato-ku, Tokyo, Japan) equipped with a CCD camera (Spot Xplorer, Monterey Imaging Technology, FL, USA) and a Fluor $20 \times$ objective lens ( $0.75 \mathrm{NA}$; Nikon), controlled by the software MetaFluor (Universal Imaging, Sunnyvale, CA, USA). The Xenon lamp (Hamamatsu, Shizuoka Prefecture, Japan) was used to provide an excitation at 
$340 \mathrm{~nm}$ and emission was collected at $460 \mathrm{~nm}$. The MQAE fluorescence intensity (F) was expressed as $\mathrm{F}=\mathrm{Fo} / \mathrm{Fcl}$, where $\mathrm{Fcl}$ was the fluorescence signal of cells recorded in normal Margo solution. The Fo was determined by Fo $=$ Fmax - Fmin.

Whole-cell patch-clamp recording. Ionic current was recorded with Axopatch-200B amplifier (Axon Instruments, Foster City, CA, USA). The command voltage was controlled by pClamp Version 9 software (Molecular Devices, Sunnyvale, CA, USA). Glass Patch pipettes were pulled on a micropipette puller (P-97, Sutter Instrument, Novato, CA, USA) to a resistance of 3-4 MQ after filled with pipette solution. The pipette solution contained (in mM): $140 \mathrm{CsCl}, 5 \mathrm{NaCl}, 2$ Mg-ATP, 10 EGTA, 10 Hepes (pH =7.2) and $110 \mathrm{U} / \mathrm{ml}$ of PKA catalytic subunit. The bath solution contained (in $\mathrm{mm}$ ): $137 \mathrm{NaCl}, 5 \mathrm{KC1}, 2 \mathrm{CaCl}_{2}, 2 \mathrm{MgCl}_{2}, 10$ Hepes and 5 glucose $(\mathrm{pH}=7.3)$. The osmolality of the bath solution and pipette solution were adjusted to $310 \mathrm{mOsm} / \mathrm{kg}$ and $290 \mathrm{mOsm} / \mathrm{kg}$ with D-mannitol, respectively. All patch-clamp recordings were made at room temperature $\left(22^{\circ} \mathrm{C}\right)$.

FACS analysis. mESCs were trypsinized and re-suspended in PBS containing $1 \%$ bovine serum albumin. Antibodies were then added and incubated for $15 \mathrm{~min}$ at room temperature in the dark. After twice washes, labeled cells were analyzed by a FACS caliber flow cytometer (Becton Dickinson, NJ, USA). A minimum of 10000 events was collected and acquired using CellQuest software (Becton Dickinson). Data were analyzed using the BD FACSDiva software. The antibodies used including SSEA1 (1/50, Millipore MAB4301). Flow cytometry studies of apoptosis were completed following standard protocols for Annexin V-FITC and PI staining kit (Life Technologies, V13421).

Teratoma formation. In all, $1 \times 10^{7}$ WT and CFTR KO mESCs were washed with $1 \times$ PBS and harvested with $0.25 \%$ trypsin (Gibco Life Technologies, Carlsbad, CA, USA). The cells were then suspended in $1 \times$ PBS (the final concentration is $2 \times 10^{7} \mathrm{mESCs} / \mathrm{ml}$ in total $500 \mu \mathrm{l}$ mixture) and injected subcutaneously into the nude mice. Four nude mice were used to inject wild-type and KO mESCs, each nude mouse was injected 4 spots, 2 spots for WT mESCs on one side and 2 spots for $\mathrm{KO} \mathrm{mESC}$ on the other side and injected $2 \times 10^{6} \mathrm{mESC} / \mathrm{spot}$. Four weeks after injection, the nude mice were killed and tumors were harvested. Teratomas were homogenized for protein and total RNA extraction or fixed in 4\% PFA overnight, and then imbedded in paraffin, sectioned and stained with hematoxylin and eosin for histological analysis.

Knockdown and overexpression in Xenopus laevis. In the loss-offunction analysis, antisense MO oligonucleotide against Xenopus CFTR RNA containing the sequence $5^{\prime}$-CTTTCTCCAGCGGCGTCTTCTGCAT-3' (CFTR MO) or a standard control MO oligo containing the sequence $5^{\prime}$-CCTCTTACCTC AGTTACAATTTATA-3' (control) was injected into the embryos at two-cell stage. To rescue the developmental defect caused by CFTR MO, the $\mathrm{MO}$ was co-injected with either CFTR or $\beta$-catenin mRNA to overexpress CFTR or $\beta$-catenin. These plasmids have been used in the experiments: full-length Xenopus $\beta$-catenin was introduced into the EcoRI restriction site of the PSP36T vector and full-length human CFTR was introduced into the BstB1 and Snab1 restriction site of the pCS2+ vector. To prepare $\mathrm{mRNA}$, the expression vector was first linearized by restriction endonuclease and then purified with PCR purification kit. The capped MRNA was synthesized in vitro using mMESSAGE mMACHINE SP6 kit (Ambion, Waltham, MA, USA, AM1340). mRNA was purified by RNA clean-up kit (Qiagen, Valencia, CA, USA, 74204).

Luciferase reporter assay. Xenopus embryos were injected at the two-cell stage two times with a mixture of $100 \mathrm{pg} \beta$-catenin/TCF reporter construct and $10 \mathrm{pg}$ pRL-CMV (Renilla luciferase). Four embryos in stage 12.5 were lysed in $40 \mu \mathrm{l}$ passive lysis buffer (Promega, Madison, WI, USA) and cleared by centrifugation. Luciferase activity was measured from $20 \mu \mathrm{l}$ extract using the Dual-Luciferase Reporter Assay System (Promega, cat\# E1910) and the LB 96V MicroLumat Plus (EG\&G Berthold Technologies, Bad Wildbad, Germany).

Statistical analysis. Data were expressed as the mean \pm S.E.M. Differences in measured variables between two groups were assessed by Student's $t$-tests. One-way ANOVA and Tukey's post hoc test were applied when there were more than two groups. Results were considered statistically significant at $P<0.05$.

\section{Conflict of Interest}

The authors declare no conflict of interest.
Acknowledgements. This study was funded by National 973 Projects, grant numbers 2012 CB944903 and 2013CB967401; National Science Foundation of China, grant numbers 31071019 and 31140034; Focused Investment Scheme of the Chinese University of Hong Kong; and Hong Kong University Grants Committee, grant numbers GRF/CUHK/466413 and 14119516.

1. Collins FS. Cystic fibrosis: molecular biology and therapeutic implications. Science 1992; 256: 774-779.

2. Tsui LC, Buchwald M, Barker D, Braman JC, Knowlton R, Schumm JW et al. Cystic fibrosis locus defined by a genetically linked polymorphic DNA marker. Science 1985; 230: 1054-1057.

3. Paccou J, Fardellone P, Cortet B. Cystic fibrosis-related bone disease. Curr Opin Pulmonary Med 2013; 19: 681-686.

4. Chen H, Ruan YC, Xu WM, Chen J, Chan HC. Regulation of male fertility by CFTR and implications in male infertility. Hum Reprod Update 2012; 18: 703-713.

5. Cohen JC, Larson JE, Killeen E, Love D, Takemaru K. CFTR and Wnt/beta-catenin signaling in lung development. BMC Dev Biol 2008; 8: 70.

6. McCallum TJ, Milunsky JM, Cunningham DL, Harris DH, Maher TA, Oates RD. Fertility in men with cystic fibrosis: an update on current surgical practices and outcomes. Chest 2000; 118: 1059-1062.

7. Hodges CA, Grady BR, Mishra K, Cotton CU, Drumm ML. Cystic fibrosis growth retardation is not correlated with loss of Cftr in the intestinal epithelium. Am J Physiol Gastrointest Liver Physiol 2011; 301: G528-G536.

8. Gee HY, Noh SH, Tang BL, Kim KH, Lee MG. Rescue of DeltaF508-CFTR trafficking via a GRASP-dependent unconventional secretion pathway. Cell 2011; 146: 746-760.

9. Tizzano EF, Chitayat D, Buchwald M. Cell-specific localization of CFTR mRNA shows developmentally regulated expression in human fetal tissues. Hum Mol Genet 1993; 2 . 219-224.

10. Levinson-Dushnik M, Benvenisty N. Involvement of hepatocyte nuclear factor 3 in endoderm differentiation of embryonic stem cells. Mol Cell Biol 1997; 17: 3817-3822.

11. Lu YC, Chen H, Fok KL, Tsang LL, Yu MK, Zhang XH et al. CFTR mediates bicarbonatedependent activation of miR-125b in preimplantation embryo development. Cell Res 2012; 22: $1453-1466$.

12. Boiani M, Scholer HR. Regulatory networks in embryo-derived pluripotent stem cells Nat Rev Mol Cell Biol 2005; 6: 872-884.

13. van Amerongen $R$, Nusse R. Towards an integrated view of Wnt signaling in development. Development 2009; 136: 3205-3214.

14. Nusse $\mathrm{R}$, Varmus $\mathrm{H}$. Three decades of Wnts: a personal perspective on how a scientific field developed. EMBO J 2012; 31: 2670-2684.

15. Lyashenko N, Winter M, Migliorini D, Biechele T, Moon RT, Hartmann C. Differential requirement for the dual functions of beta-catenin in embryonic stem cell self-renewal and germ layer formation. Nat Cell Biol 2011; 13: 753-761.

16. Anton R, Kestler HA, Kuhl M. Beta-catenin signaling contributes to stemness and regulates early differentiation in murine embryonic stem cells. FEBS Lett 2007; 581: 5247-5254.

17. ten Berge D, Kurek D, Blauwkamp T, Koole W, Maas A, Eroglu E et al. Embryonic stem cells require Wnt proteins to prevent differentiation to epiblast stem cells. Nat Cell Biol 2011; 13 : 1070-1075.

18. Ying QL, Wray J, Nichols J, Batlle-Morera L, Doble B, Woodgett J et al. The ground state of embryonic stem cell self-renewal. Nature 2008; 453: 519-523.

19. Sato N, Meijer L, Skaltsounis L, Greengard P, Brivanlou AH. Maintenance of pluripotency in human and mouse embryonic stem cells through activation of Wnt signaling by a pharmacological GSK-3-specific inhibitor. Nat Med 2004; 10: 55-63.

20. Aramaki S, Hayashi K, Kurimoto $\mathrm{K}$, Ohta $\mathrm{H}$, Yabuta $\mathrm{Y}$, Iwanari $\mathrm{H}$ et al. A mesodermal factor, $\mathrm{T}$, specifies mouse germ cell fate by directly activating germline determinants. Dev Cell 2013; 27: $516-529$.

21. Naito AT, Shiojima I, Akazawa H, Hidaka K, Morisaki T, Kikuchi A et al. Developmental stagespecific biphasic roles of Wnt/beta-catenin signaling in cardiomyogenesis and hematopoiesis. Proc Natl Acad Sci USA 2006; 103: 19812-19817.

22. Umehara H, Kimura T, Ohtsuka S, Nakamura T, Kitajima K, Ikawa M et al. Efficient derivation of embryonic stem cells by inhibition of glycogen synthase kinase-3. Stem cells 2007; 25: 2705-2711.

23. Kiyonari H, Kaneko M, Abe S, Aizawa S. Three inhibitors of FGF receptor, ERK, and GSK3 establishes germline-competent embryonic stem cells of C57BL/6N mouse strain with high efficiency and stability. Genesis 2010; 48: 317-327.

24. Gadue P, Huber TL, Paddison PJ, Keller GM. Wnt and TGF-beta signaling are required for the induction of an in vitro model of primitive streak formation using embryonic stem cells. Proc Natl Acad Sci USA 2006; 103: 16806-16811.

25. Lindsley RC, Gill JG, Kyba M, Murphy TL, Murphy KM. Canonical Wnt signaling is required for development of embryonic stem cell-derived mesoderm. Development 2006; 133 3787-3796.

26. Kemp C, Willems E, Abdo S, Lambiv L, Leyns L. Expression of all Wnt genes and their secreted antagonists during mouse blastocyst and postimplantation development. Dev Dyn 2005; 233: 1064-1075. 
27. Pankow S, Bamberger C, Calzolari D, Martínez-Bartolomé S, Lavallée-Adam M, Balch WE et al. F508 CFTR interactome remodelling promotes rescue of cystic fibrosis. Nature 2015; 528: $510-516$.

28. Sun TT, Wang Y, Cheng H, Xiao HZ, Xiang JJ, Zhang JT et al. Disrupted interaction between CFTR and AF-6/afadin aggravates malignant phenotypes of colon cancer. Biochimica Et Biophys Acta 2014; 1843: 618-628.

29. Ruan YC, Wang Y, Da Silva N, Kim B, Diao RY, Hill E et al. CFTR interacts with ZO-1 to regulate tight junction assembly and epithelial differentiation through the ZONAB pathway. J Cell Sci 2014; 127: 4396-4408.

30. Hoppler S. Studying Wnt signaling in Xenopus. Methods Mol Biol 2008; 469: 319-332.

31. Schohl A, Fagotto F. A role for maternal beta-catenin in early mesoderm induction in Xenopus. EMBO J 2003; 22: 3303-3313.

32. Dush MK, Martin GR. Analysis of mouse Evx genes: Evx-1 displays graded expression in the primitive streak. Developmental biology 1992; 151: 273-287.

33. Robb L, Tam PP. Gastrula organiser and embryonic patterning in the mouse. Semin Cell Dev Biol 2004; 15: 543-554.

34. Moon RT, Campbell RM, Christian JL, McGrew LL, Shih J, Fraser S. Xwnt-5A: a materna Wnt that affects morphogenetic movements after overexpression in embryos of Xenopus laevis. Development 1993; 119: 97-111.

35. Moon RT, DeMarais A, Olson DJ. Responses to Wnt signals in vertebrate embryos may involve changes in cell adhesion and cell movement. J Cell Sci 1993; 17: 183-188.
36. Heasman J, Crawford A, Goldstone K, Garner-Hamrick P, Gumbiner B, McCrea P et al. Overexpression of cadherins and underexpression of beta-catenin inhibit dorsal mesoderm induction in early Xenopus embryos. Cell 1994; 79: 791-803.

37. Nichols D, Chmiel J, Berger M. Chronic inflammation in the cystic fibrosis lung: alterations in inter- and intracellular signaling. Clin Rev Allergy Immunol 2008; 34: 146-162.

38. Dong ZW, Chen J, Ruan YC, Zhou T, Chen Y, Chen Y et al. CFTR-regulated MAPK/NFkappaB signaling in pulmonary inflammation in thermal inhalation injury. Sci Rep 2015; 5: 15946.

39. Le Henaff C, Faria Da Cunha M, Hatton A, Tondelier D, Marty C, Collet C et al. Genetic deletion of keratin 8 corrects the altered bone formation and osteopenia in a mouse model of cystic fibrosis. Hum Mol Genet 2016; 25: 1281-1293.

40. Le Henaff C, Mansouri R, Modrowski D, Zarka M, Geoffroy V, Marty C et al. Increased NF-kappaB activity and decreased Wnt/beta-catenin signaling mediate reduced osteoblast differentiation and function in DeltaF508 cystic fibrosis transmembrane conductance regulator CFTR) mice. J Biol Chem 2015; 290: 18009-18017.

41. Snouwaert JN, Brigman KK, Latour AM, Malouf NN, Boucher RC, Smithies 0 et al. An animal model for cystic fibrosis made by gene targeting. Science 1992; 257: 1083-1088.

42. Shi W, Xu G, Wang C, Sperber SM, Chen Y, Zhou Q et al. Heat shock 70-kDa protein 5 Hspa5) is essential for pronephros formation by mediating retinoic acid signaling. J Biol Chem 2015; 290: 577-589.

43. Gordon R, Bjorklund NK, Nieuwkoop PD. Appendix: dialogue on embryonic induction and differentiation waves. Int Rev Cytol 1994; 150: 373-420.

\section{Supplementary Information accompanies this paper on Cell Death and Differentiation website (http://www.nature.com/cdd)}

